\title{
Carbon-rich giants in the HR diagram and their luminosity function $\star, \star \star, \star \star \star$
}

\author{
J. Bergeat, A. Knapik, and B. Rutily
}

Centre de Recherche Astronomique de Lyon (UMR 5574 du CNRS), Observatoire de Lyon, 9 avenue Charles André, 69561 St-Genis-Laval Cedex, France

Received 3 January 2002 / Accepted 15 March 2002

\begin{abstract}
The luminosity function (LF) of nearly 300 Galactic carbon giants is derived. Adding BaII giants and various related objects, about 370 objects are located in the RGB and AGB portions of the theoretical HR diagram. As intermediate steps, (1) bolometric corrections are calibrated against selected intrinsic color indices; (2) the diagram of photometric coefficients $\langle k\rangle^{1 / 2}$ vs. astrometric true parallaxes $\varpi$ are interpreted in terms of ranges of photospheric radii for every photometric group; (3) coefficients $C_{R}$ and $C_{L}$ for bias-free evaluation of mean photospheric radii and mean luminosities are computed.

The LF of Galactic carbon giants exhibits two maxima corresponding to the HC-stars of the thick disk and to the CV-stars of the old thin disk respectively. It is discussed and compared to those of carbon stars in the Magellanic Clouds and Galactic bulge. The HC-part is similar to the LF of the Galactic bulge, reinforcing the idea that the Bulge and the thick disk are part of the same dynamical component. The CV-part looks similar to the LF of the Large Magellanic Cloud (LMC), but the former is wider due to the substantial errors on HIPPARCOS parallaxes.

The obtained mean luminosities increase with increasing radii and decreasing effective temperatures, along the HC-CV sequence of photometric groups, except for HC0, the earliest one. This trend illustrates the RGB- and AGB-tracks of low- and intermediate-mass stars for a range in metallicities. From a comparison with theoretical tracks in the HR diagram, the initial masses $M_{\mathrm{i}}$ range from about 0.8 to $4.0 M_{\odot}$ for carbon giants, with possibly larger masses for a few extreme objects. A large range of metallicities is likely, from metal-poor HC-stars classified as $\mathrm{CH}$ stars on the grounds of their spectra (a spheroidal component), to near-solar compositions of many $\mathrm{CV}$-stars. Technetium-rich carbon giants are brighter than the lower limit $M_{\text {bol }} \simeq-3.6 \pm 0.4$ and centered at $\left\langle M_{\text {bol }}\right\rangle \simeq-4.7_{-0.9}^{+0.6}$ at about $\left\langle T_{\text {eff }}\right\rangle \simeq(2935 \pm 200) \mathrm{K}$ or CV3-CV4 in our classification. Much like the results of Van Eck et al. (1998) for S stars, this confirms the TDU-model of those TP-AGB stars. This is not the case of the HC-stars in the thick disk, with $\left\langle T_{\text {eff }}\right\rangle \gtrsim 3400 \mathrm{~K}$ and $\left\langle M_{\text {bol }}\right\rangle \gtrsim-3.4$. The faint HC1 and HC2-stars $\left(\left\langle M_{\text {bol }}\right\rangle \simeq-1.1_{-1.0}^{+0.7}\right)$ are found slightly brighter than the BaII giants $\left(\left\langle M_{\mathrm{bol}}\right\rangle \simeq-0.3 \pm 1.3\right)$ on average. Most RCB variables and HdC stars range from $M_{\mathrm{bol}} \simeq-1$ to -4 against -0.2 to -2.4 for those of the three population II Cepheids in the sample. The former stars show the largest luminosities $\left(\left\langle M_{\mathrm{bol}}\right\rangle \lesssim-4\right.$ at the highest effective temperatures $(6500-7500 \mathrm{~K})$, close to the $M_{\mathrm{bol}} \simeq-5$ value for the hot LMC RCB-stars (W Men and HV 5637). A full discussion of the results is postponed to a companion paper on pulsation modes and pulsation masses of carbon-rich long period variables (LPVs; Paper IV, present issue).
\end{abstract}

Key words. stars: AGB and post-AGB - stars: carbon - stars: late-type - stars: fundamental parameters stars: Hertzsprung-Russell (HR) and C-M diagrams - stars: luminosity function, mass function

\section{Introduction}

The carbon-rich giants are stars of low and intermediate initial masses (LMS, IMS) evolved far from the main sequence in the Hertzsprung-Russell (HR) diagram, up to the red giant region.

Send offprint requests to: J. Bergeat,

e-mail: bergeat@obs.univ-lyon1.fr

* This research has made use of the Simbad database operated at CDS, Strasbourg, France.

$\star \star$ Partially based on data from the ESA HIPPARCOS astrometry satellite.

$\star \star \star$ Table 2 is only available in electronic form at the CDS via anonymous ftp to cdsarc.u-strasbg.fr $(130.79 .128 .5)$ or via http://cdsweb.u-strasbg.fr/cgi-bin/qcat?J/A+A/390/967
Modified surface chemical compositions are observed, including enhanced carbon to oxygen $(\mathrm{C} / \mathrm{O})$ ratios, up to $\mathrm{C} / \mathrm{O} \geq 1$. Products of thermonuclear reactions $(\mathrm{C}$, s-process elements, etc.) are obviously dragged up to the surface by convection reaching layers of processed material. The study of carbon giants is important in itself, but also due to the matter they return to interstellar medium through mass loss, with rates in the $10^{-8}-10^{-4} M_{\odot} \mathrm{yr}^{-1}$ range (e.g. Schöier \& Olofsson 2001 and references therein). The derivation of luminosities of Galactic carbon stars was for a long time hampered by missing usable parallaxes, and various methods were used (e.g. Alksne et al. 1991; Sect. 8.2, p. 107), before the HIPPARCOS mission (ESA 1997). Analyses of part of the HIPPARCOS data on carbon 
stars has been published (Alksnis et al. 1998; Wallerstein \& Knapp 1998; Knapp et al. 2001; Mennessier et al. 2001). A similar study has been performed for S stars (Van Eck et al. 1998). Conversely, the study of the evolved carbon giants was developed since the eighties in nearby galaxies of the Local Group: see e.g. the reviews of Azzopardi (1999a, 1999b, 2000), and Azzopardi et al. (1999). A common distance modulus is assumed for every star in a given system, and apparent magnitudes are used to establish HR diagrams and luminosity functions (LFs). The Large Magellanic Cloud (LMC) and Small Magellanic Cloud (SMC) were thoroughly studied, but close dwarf systems were explored as well. The more distant M 31 was reached with 31 carbon stars detected (Brewer et al. 1995). Luminosity functions and loci in the HR diagram, were obtained for carbon stars in nearby systems and in the Galactic bulge (Westerlund et al. 1991, 1992, 1995; Azzopardi et al. 1991).

Carbon giants may span a more or less wide range, from $M_{\text {bol }} \simeq 0$ or -1 to $M_{\text {bol }} \simeq-6$ or -7 at most, with one (or two maximum), at $M_{\text {bol }} \simeq-1$ or -2 (eventually), and $M_{\text {bol }} \simeq-4$ or -5 respectively. Decreasing bolometric magnitudes (increasing luminosities) are correlated with decreasing effective temperatures in the 6000-2000 K range. Thus, the carbon giants populate inclined strips in the HR diagrams of those extragalactic systems. Those loci however shift toward higher effective temperatures and larger luminosities for lower initial metallicities (e.g. Westerlund et al. 1995 for SMC: $Z=0.004$ typically).

These observations are at least partly consistent with evolved models of LMS and IMS (e.g. the reviews of Iben \& Renzini 1983 and Busso et al. 1999). The bright carbon giants are found on the asymptotic giant branch (AGB), especially the region where models experience thermal pulses (TPs), i.e. temporal He-shell ignitions (TP-AGB). After a more or less high number of TPs, they may become carbon-rich, provided $M_{\mathrm{i}} \lesssim 4 M_{\odot}$, since at larger masses, hot bottom burning (HBB, i.e. transformation of ${ }^{12} \mathrm{C}$ into ${ }^{13} \mathrm{C}$ and then ${ }^{14} \mathrm{~N}$ ) prevents $C / O \geq 1$ to be reached (e.g. Marigo et al. 1999). Mass loss can however reduce the envelope mass and stop HBB while thermal pulses are still ongoing (Frost et al. 1998). Stars with initial masses larger than $4 M_{\odot}$ could thus turn temporarily to carbon-rich objects embedded in thick circumstellar shells, a consequence of strong mass loss. The low-luminosity carbon giants $\left(M_{\text {bol }} \gtrsim-3.5\right)$ however fail to be explained by standard models (e.g. Marigo et al. 1996, 1999, and references therein).

An alternative explanation for anomalous surface abundances may be past mass exchange in a binary system, where a TP-AGB companion became a white dwarf (Han et al. 1995 and references therein). This is the currently-accepted model for BaII stars, many of them having proved to be binary members (Mc Clure et al. 1980; McClure 1984; Jorissen \& Boffin 1992). The low-luminosity carbon stars classified into the Rtypes however fail to show any evidence of binarity, and Mc Clure (1997a) considered they could result from coalescence of components in a former binary system.

From the data collected in several star clusters in the LMC, Bessell et al. (1981, 1983) found a sharp transition from oxygen stars to carbon stars at $M_{\text {bol }} \simeq-4.3$ and $T_{\text {eff }} \simeq 3670 \mathrm{~K}$. The fainter and hotter carbon giants (i.e. counterparts of HC-stars in the Galaxy) were not found in those clusters, and other LMCfields as well (see data from Costa \& Frogel 1996). Such stars were observed in the SMC (e.g. Westerlund et al. 1995) and in the Galactic bulge (Rich 1989). Bessell et al. deduced that the minimum initial mass for obtaining carbon stars is about $0.9 M_{\odot}$ at $[\mathrm{Fe} / \mathrm{H}] \simeq-1$ and $1.3 M_{\odot}$ at $[\mathrm{Fe} / \mathrm{H}] \simeq 0$. The cluster diagrams and specially the turn-off of NGC 121 aged $10.5 \mathrm{Gyr}$ where no carbon star was observed, were used to produce those estimates.

The kinematics of carbon $N$ stars we mostly classified into the CV-groups, being similar to that of F5 dwarfs (Dean 1976), the deduced equivalent mass is about $1.4 M_{\odot}$. The carbon $\mathrm{R}$ stars we mostly classified into the HC-groups, correspond to GK dwarfs (Mc Leod 1947), pointing to main sequence masses smaller than $1 M_{\odot}$. The HC-stars which are $\mathrm{CH}$ stars on spectroscopic grounds, are halo tracers (Hartwick \& Cowley 1985) and their initial masses should not exceed $0.8 M_{\odot}$.

From an investigation of a flux-limited sample of Galactic carbon stars taken from the Two Micron Sky Survey (TMSS 1969), Claussen et al. (1987) found that their main-sequence progenitors have masses between 1.2 and $1.6 M_{\odot}$, i.e. should be F-type dwarfs. Thronson et al. (1987) used the IRAS Point Source Catalogue (IRAS 1988) to study a flux-limited sample of highly-evolved carbon-rich and oxygen-rich stars in the Milky Way. They found a local birthrate for their carbon stars equivalent to the deathrate for stars that leave the main sequence with masses in the $3-5 M_{\odot}$ range. This is consistent with the wavelength ranges of the used catalogues, resp. TMSS $(2.2 \mu \mathrm{m})$ and IRAS $(12,25,60,100 \mu \mathrm{m})$. Zuckerman et al. (1986) presented evidence that carbon-rich stars which have circumstellar envelopes with large "terminal" outflow velocities $\left(V_{\infty}\right)$, are preferentially located close to the galactic plane (low $|b|$ used instead of $|z|$, which may generate some confusion). This class of carbon stars which lies at $|b| \leq 10^{\circ}$, has outflow velocities $V_{\infty} \gtrsim 18 \mathrm{~km} \mathrm{~s}^{-1}$ (Zuckerman \& Dyck 1989). Large masses $\left(M_{\mathrm{ms}} \geq 3 M_{\odot}\right)$ were proposed for those objects, while a range of $1 \leq M \leq 3 M_{\odot}$ was suggested for $-9 \lesssim V_{\infty} \lesssim 18 \mathrm{~km} \mathrm{~s}^{-1}$. A $107 \mathrm{pc}$ scale height was obtained for the stars with high outflow velocities, a value typical of main-sequence stars with masses larger than 2.5-4 $M_{\odot}$ (Barnbaum et al. 1991). These results imply that the range of initial main-sequence masses, is fairly large for carbon giants. In their investigations of TMSS and IRAS carbon stars, Claussen et al. (1987) and Thronson et al. (1987) actually delineated different subsamples of CV-stars.

Making use of the true parallaxes as estimated by Knapik el al. (1998) from HIPPARCOS data, we investigate in the present paper, the LF and loci of about 370 carbon and BaII giants of the Galactic disk, in the HR diagram. The Lutz-Kelker bias being taken into account, those parallaxes are intended for statistical purposes. The effective temperatures and bolometric apparent magnitudes of Paper I extended to a larger sample, will be used hereafter. The evaluation of the pulsation masses of carbon long period variables (LPVs) and of the mass-luminosity diagram is postponed to a companion paper (Bergeat et al. 2002b, hereafter Paper IV).

The data of Bergeat et al. (2001, hereafter Paper I) and references therein, and Bergeat et al. (2002a, hereafter Paper II), 
are summarized and extended in Sect. 2 through the calibration of bolometric corrections (Table A.1 in appendix) to be applied to additional stars from the HIPPARCOS sample (not considered in Paper I because of incomplete SEDs). Then the coefficients $C_{\mathrm{R}}$ and $C_{\mathrm{L}}$ are introduced in Sect. 3, to allow derivation of unbiased mean photospheric radii and luminosities respectively. The corresponding data for individual stars are given in Table 2 (only available in electronic form at CDS), specifically effective temperatures and absolute bolometric magnitudes.

The diagrams of relative angular diameters $\langle k\rangle^{1 / 2}$ from photometry vs. estimated true parallaxes $\varpi$ from HIPPARCOS astrometry, are presented for the different photometric groups. They are interpreted in terms of ranges in photospheric radii for each photometric group. Mean values and ranges (effective temperatures, photospheric radii and luminosities) were computed for the fourteen photometric groups of carbon giants (Sect. 4). The mean bolometric magnitudes of BaII giants were computed for comparison purposes from data of Bergeat \& Knapik (1997).

The mean values according to variability classes amongst carbon giants, were also calculated (Sect. 5). The luminosity function (LF) of Galactic carbon giants in the Sun vicinity (Sect. 6), is compared to those of the Galactic bulge and of the Magellanic Clouds. The loci of carbon and BaII giants in the HR diagram are then presented (Sect. 7), and confronted to the predictions of theoretical models of stellar evolution. The various star categories are discussed (BaII, HC- and CVgiants, RCB variables, HdC giants and carbon-rich cepheids). The presence of Technetium is discussed, and the ranges in initial masses $\left(M_{\mathrm{i}}\right)$ inferred. The results are briefly summarized in Sect. 8, and a full discussion is postponed to Paper IV mainly devoted to pulsation modes and pulsation masses of carbonrich long period variables (LPVs).

\section{The photometric data}

\subsection{Photometric groups and populations}

The first classification of the carbon-rich giants in discrete photometric groups ( 13 groups named $\mathrm{HC} i$ and $\mathrm{CV} j$ with $i=0$ to 5 and $j=1$ to 7 , and the fourteenth SCV as a transition to bright S stars), was established by Knapik \& Bergeat (1997), Bergeat et al. (1999) and Knapik et al. (1999). It relies on the intrinsic spectral energy distributions (SEDs) of those stars from UV to IR $(0.36$ to $25 \mu \mathrm{m})$ which were gathered into discrete groups. A sequence of 13 groups was obtained from the earliest one to the latest one, completed by a fourtheenth one (SCV) corresponding to the spectroscopic SC variables which is a transition to $\mathrm{S}$ stars. The mean SEDs exhibit more or less regularly increasing color indices and the eighteen best-determined ones were selected and settled to a zero-magnitude at $1.08 \mu \mathrm{m}$. The name "carbon variable" (CV) was initially selected because the members of all those later seven groups (CV1 to CV7) are variable stars or "suspected new variables". On the contrary, a majority of the members of the six earlier groups are constant in light, i.e. non-variable stars as confirmed by repeated high-accuracy HIPPARCOS photometric measurements (ESA 1997). A few irregulars and small-amplitude variables, carbon cepheids and RCB-variables are also present. Those earlier groups were named "hot carbon" ( $\mathrm{HC}$, from $\mathrm{HC} 0$ to HC5).

The interstellar and circumstellar extinction on the line of sight to each star is simultaneously determined, and a mean coefficient $\langle k\rangle^{1 / 2}$ calculated, which proved to be an angular diameter on a relative scale for a given effective temperature (or eventually for a given photometric group: see Paper I). The new effective temperatures proposed for carbon-rich giants and related objects in Paper I rely on spectral energy distributions (SEDs), model atmospheres and measured angular diameters. The classification and the homogeneous temperature scale obtained proved to be tightly correlated. It was concluded that the main parameter of this photometric classification is effective temperature (Paper I), which is not the case of classical spectral classifications. Amongst the remaining parameters, the $\mathrm{C} / \mathrm{O}$ ratio has an important influence. For $\mathrm{CV}$-stars, the mean values and dispersions increase along the sequence (CV2 to CV6) of photometric groups (Paper I, Sect. 10).

Bergeat et al. (Paper II) analyzed the space distribution and kinematics of Galactic carbon giants. They showed that the HC-sample (essentially early-R stars) is a component of the thick disk contaminated by the $\mathrm{CH}$ stars which are a spheroidal contribution (see also Hartwick \& Cowley 1985). The HC-stars not classified as $\mathrm{CH}$ stars probably correspond to old $(\simeq 11 \mathrm{Gyr})$ low initial-mass stars (say $\leq 1.1 M_{\odot}$ ). It was also shown in Paper II that the CV-sample (mainly $N$ stars) is a component of the old (thin) disk, dated $(3 \pm 1)$ Gyr on average, but with a large spread from a few $10^{2} \mathrm{Myr}$ to nearly $10-12 \mathrm{Gyr}$. The corresponding initial masses should range from about $1 M_{\odot}$ to a few solar masses. Corrections to the (small) effects of the Malmquist bias on the HIPPARCOS sample of carbon giants were also obtained in Paper II. They are applied hereafter to quoted mean luminosities.

\subsection{The calibration of bolometric corrections}

Effective temperatures were derived for about 390 carbon-rich giants whose apparent bolometric magnitudes were deduced as well (Paper I). The used method makes use of both the whole integrated deredenned SED ("bolometric" value) and calibrated color indices ("spectral" value). About 220 carbon stars observed by the satellite HIPPARCOS (ESA 1997) were involved in this study. There remained 81 HIPPARCOS carbon stars $(82$ SEDs) with SEDs partially documented. The "spectral" method was the only one available for effective temperature evaluation of those latter stars. Their apparent bolometric magnitudes were estimated from calibrations of various bolometric corrections $B C_{\mathrm{m}(\lambda)}$ in terms of several (intrinsic) color indices $I C_{0}$.

The calibrations were acquired from the sample of 390 stars (Paper I). Several couples $(B C ; I C)$ were finally selected by trial and error amongst our data. They represent our "best choices" on the $V(0.55 \mu \mathrm{m})$ to $K(2.2 \mu \mathrm{m})$ range. The classical $C B_{K}$ vs. $[J-K]_{0}$ plot is shown in Fig. 1. The earlier diagram of Frogel et al. (1980; their Fig. 2, p. 498) is confirmed and extended at both ends. 


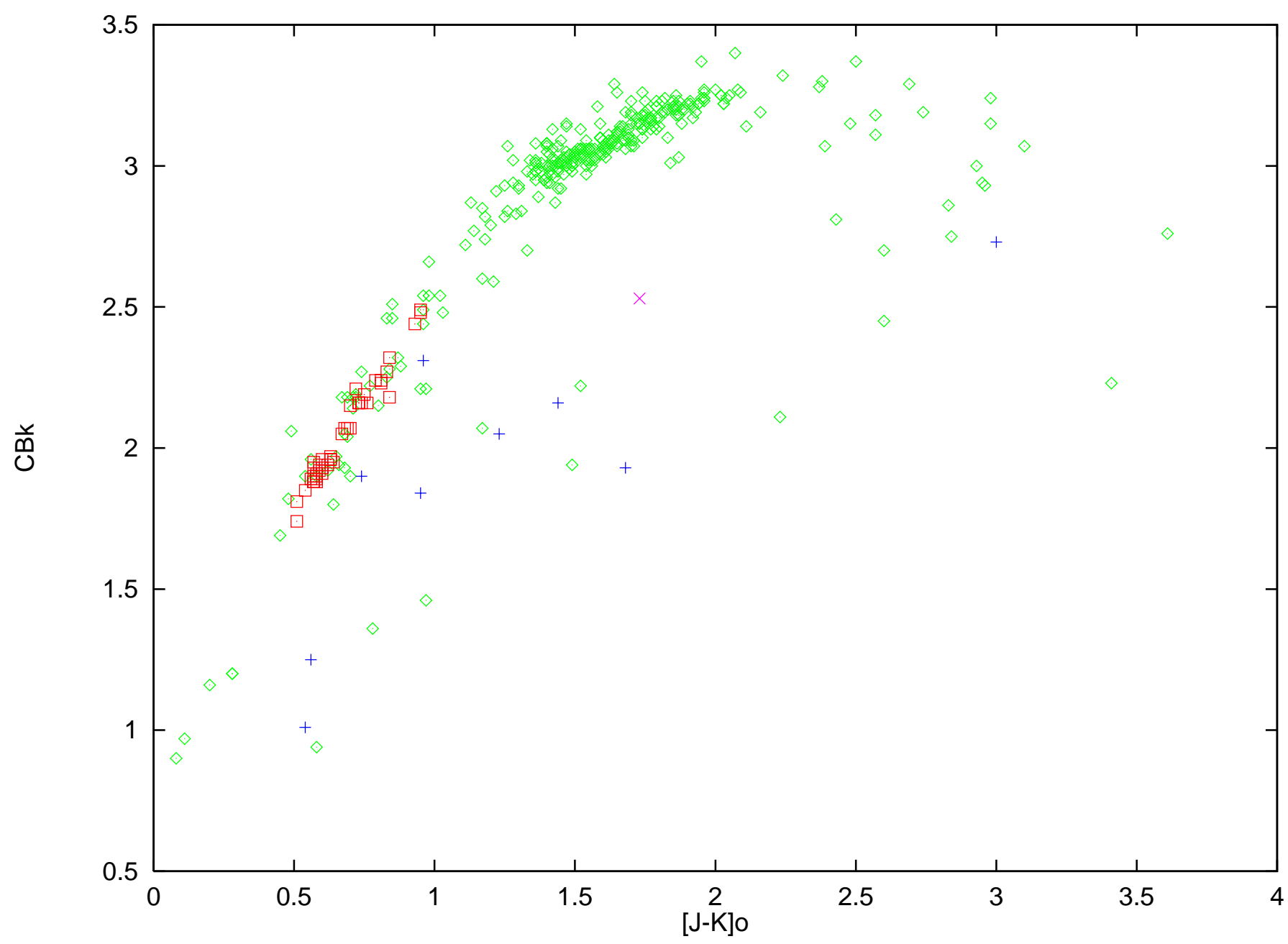

Fig. 1. The $C B_{K}$ vs. $[J-K]_{0}$ diagram for 375 red giants: 315 carbon stars of the SCV, HC and CV-groups (diamond-shaped symbols; 1 with "silicate" IR excess: X-symbol), 15 RCB variables (ox.-groups; crosses), 45 Ba II stars (ox.-groups; squares).

Except for about $20-30$ objects, the stars populate a welldefined locus from $(0.0,0.8)$ to $(2.5,3.4)$. The discrepant objects are located below this locus due to thermal emission from circumstellar (CS) grains in the infrared, usually starting from the $H$-bandpass at $1.65 \mu \mathrm{m}$ and increasing longwards. The $J$-bandpass at $1.25 \mu \mathrm{m}$ is much less affected while the $K$ bandpass at $2.2 \mu \mathrm{m}$ is much more affected. Finally the CS vector in the diagram is oriented toward lower $C B_{K}$ and larger $[J-K]_{0}$ as seen in Fig. 1. The extreme ("infrared") carbon stars such as C2619 = IRC $+10216=\mathrm{CW}$ Leo and C2724 = CIT6 = RW LMi, are so strongly shifted that they fall outside the frame. In this diagram, the Ba II stars do not differ appreciably from the hot carbon (HC) stars, which allows one to adopt the same mean relation. With the exception of a few stars, the RCB variables do exhibit a substantial IR excess. They populate a strip located well below the relation for carbon and Ba II stars. This is also the case of C2011, a star with a silicate-type IR excess. In such cases the $C B_{K}$ vs. $[J-K]_{0}$ couple cannot be used to estimate bolometric corrections. For $[J-K]_{0} \geq 2.1$, that is dealing with carbon variables of the CV6-CV7 groups, the proportion of stars with an appreciable IR excess increases. The locus widens in a leek-shaped structure for increasing color indices. Following a wide maximum, a decrease of the bolometric correction is observed for increasing indices. Here again, the mean relation cannot safely be used. Thus, we calibrated data at shorter wavelengths. The assumption of constant total radiated power when absorbed light is reradiated in the IR by CS grains deserves discussion, depending on shell geometry and/or dust properties. Bergeat et al. (2002, Paper I, Sect. 13) found that for a few hot carbon (HC) stars and RCB variables, corrections need to be applied due to nonspherical symmetry. On the contrary, they estimated that reasonable values can be obtained from dereddened SEDs without IR excesses or from observed SEDs including IR excesses in the case of cool carbon variables (CV6-CV7) with strong thermal emission.

Finally, we made use of eight $B C_{\mathrm{m}(\lambda)}$ vs. $I C_{0}$ mean relations, namely $C B_{[0.78]}, C B_{[1.08]}, C B_{H}$ and $C B_{K}$ vs. $[V-[1.08]]_{0}, C B_{H}$ and $C B_{K}$ vs. [[1.08] $\left.-K\right]_{0}$, and finally $C B_{H}$ and $C B_{K}$ vs. $[J-K]_{0}$. The magnitudes [0.78] and [1.08] are such as defined by Knapik \& Bergeat (1997) from the data obtained in narrow bandpasses by Baumert (1972). The adopted mean relations are given in Appendix. Departures between values deduced from short wavelengths on one hand and from long wavelengths on the other hand, if any, are estimated to be less than 0.1 mag. Finally, the estimates are 
averaged and the errors on the resulting bolometric correction should not exceed 0.05 mag for the 81 carbon stars studied in this way. The absolute bolometric magnitudes in forthcoming sections are of course affected, but the effect can be neglected when true luminosity dispersions and uncertainties on true parallaxes are taken into account.

\subsection{The three encountered biases}

A detailed study of the influence of three encountered biases on averaged quantities as derived from the HIPPARCOS parallaxes was given by Knapik (1999). No appreciable bias is expected on mean HIPPARCOS proper motions. Essential parts of that work can be found in Knapik et al. (1998) and Paper II. Here, we present a summary of the effects of biases on averaged quantities.

The Malmquist bias $(1924,1936)$ affects any sample delineated on the grounds of a limited apparent magnitude. We found $V_{1} \simeq 10$ for the HIPPARCOS sample of carbon stars (Knapik et al. 1998), refined as $V_{1} \simeq 10.4$ for the HC-stars and $V_{1} \simeq 9.6$ for the CV-stars (Paper II). Mean absolute magnitudes are found too bright since increasing selection occurs amongst more and more distant stars, favoring the intrinsically brighter objects. In Sect. 4 of Paper II, corrections to mean absolute bolometric magnitudes were derived which amounted to a few tenths of a magnitude at most $(+0.32 \mathrm{mag}$ at $\mathrm{HC} 1$ and +0.2 mag at $\mathrm{HC} 2$ are the largest values, against $0.1 \mathrm{mag}$ or less for the other groups). A second aspect is the increasing deficits at increasing distances from Sun, in stellar number densities (the missing objects in Fig. 1 of Paper II) and in stellar surface densities as projected on the Plane (Sect. 5.1 of Paper II). This latter effect lowers the $q$-exponent of the HIPPARCOS distribution of true (as opposed to observed) parallaxes

$N(\varpi) \mathrm{d} \varpi \propto \varpi^{-q} \mathrm{~d} \varpi$

from about 3 for stars of $\varpi \gtrsim 1$ mas, to 2.35 on average (Knapik et al. 1998 and Sect. 2.3 of Paper II). The influence is still larger at even larger distances, but fewer HIPPARCOS carbon stars are involved.

The bias studied by Smith \& Eichhorn (1996) occurs every time a mean value is calculated for a quantity which is not a linear function of true parallaxes. Even if the distribution according to true parallaxes is a Gaussian, that of distances or absolute magnitudes is not. Smith \& Eichhorn derived the mathematical expectation of distances from parallaxes affected by arbitrarily high errors ( $\sigma$, their Fig. 1, p. 213). An overestimate is found for $\alpha=\sigma / \varpi<0.74$ which changes into an underestimate for $\alpha>0.74$. The consequence of this effect and the methods used to avoid it in various circumstances can be found in Bergeat et al. (1998), Knapik et al. (1998), Sects. 3.1 and 3.2 of Paper II, Sects. 3.1 and 3.3 of Paper III, and in Sect. 2.1 of the present paper.

The third bias results from the effect of errors on a distribution non-uniform in parallaxes. Let us assume that the probability distribution function (pdf) of repeated individual observations of a single true parallax $(\varpi)$ is a Gaussian centered on the $\varpi$-value, with $\sigma$ as a standard deviation. Now we consider an other problem which is to derive the pdf of true parallaxes $(\varpi)$ for a given sample of stars whose observed parallaxes are the $\varpi_{0}$ 's from the HIPPARCOS catalogue. This pdf is the same Gaussian as mentioned above, provided the stars are uniformly distributed in true parallaxes (Lutz \& Kelker 1973). This latter condition is completely unrealistic and Lutz \& Kelker adopted a uniform space density in their pioneering work, that is

$N(\varpi) \mathrm{d} \varpi \propto \varpi^{-4} \mathrm{~d} \varpi$.

The resulting pdf weighted by $\varpi^{-4}$ is no longer a Gaussian. The net effect when exploiting observed parallaxes is that stars are seen, on average, as closer to Sun than they are indeed. The larger the errors on observed parallaxes, the larger the bias for a given pdf. Coupled with the Smith \& Eichhorn bias on absolute magnitudes, this is the so-called Lutz-Kelker bias (Lutz \& Kelker 1973). The effect on mean values for samples of carbon giants is lower than it is in their original paper since Eq. (1) prevails on Eq. (2) for that Malmquist-biased sample whose distribution is flattened toward the Galactic plane. A flattened sample uniform in volumic density, free of the Malmquist bias, would have $q \simeq 3$. Knapik et al. (1998) proposed a calculation of the mathematical expectation of the difference $\varpi-\varpi_{0}$. They were able to model the distribution of errors on observed HIPPARCOS parallaxes of carbon stars.

It is interesting to note that future astrometric missions like GAIA (ESA 1995) will be almost free from those biases, due to their very high accuracies and extensive sampling, reaching the edges of the galactic disk. The main difficulty will then result from non-uniform brightness distributions on extended stellar discs.

\section{The C-coefficients, luminosities and radii}

\subsection{The $C_{R}$-coefficient}

In Paper I was established (Eq. (33)) the relation

$\langle k\rangle=\left(\Phi / \Phi_{0}\right)^{2}$

where $\Phi$ is the true (observed) angular diameter of the star and $\Phi_{0}$ its diameter would its magnitude be $[1.08]_{0}=0$, other thinks being equal.

The $\langle k\rangle$-coefficient is a mean value over the wavelengths used, that is derived directly from photometry (Knapik et al. 1999, Sect. 2.4). It is thus an angular diameter on a relative scale as confirmed in Paper I (Sect. 8). It correlates with astrometric data, namely estimated true parallaxes $\varpi$ from Knapik et al. (1998; Sect. 4), in the expected way. The reference angular diameters $\Phi_{0}$ were calibrated as a function of effective temperatures (Paper I, Sect. 11), viz. $\Phi_{0}\left(T_{\text {eff }}\right)$. The coefficient may still be written as

$\langle k\rangle^{1 / 2} \simeq 2.063 \times 10^{8} \frac{R}{R_{\odot}} \frac{R_{\odot}}{D} /\left(\frac{\Phi_{0}}{2}\right)_{\text {mas }}$,

where $R$ is the photospheric radius of the star. Replacing $\varpi=$ $1 / D$, we introduce a new coefficient

$C_{R}=\frac{\Phi_{0} / 2}{R / R_{\odot}} \simeq \frac{\varpi_{\mathrm{mas}}}{214.94\langle k\rangle^{1 / 2}}$ 


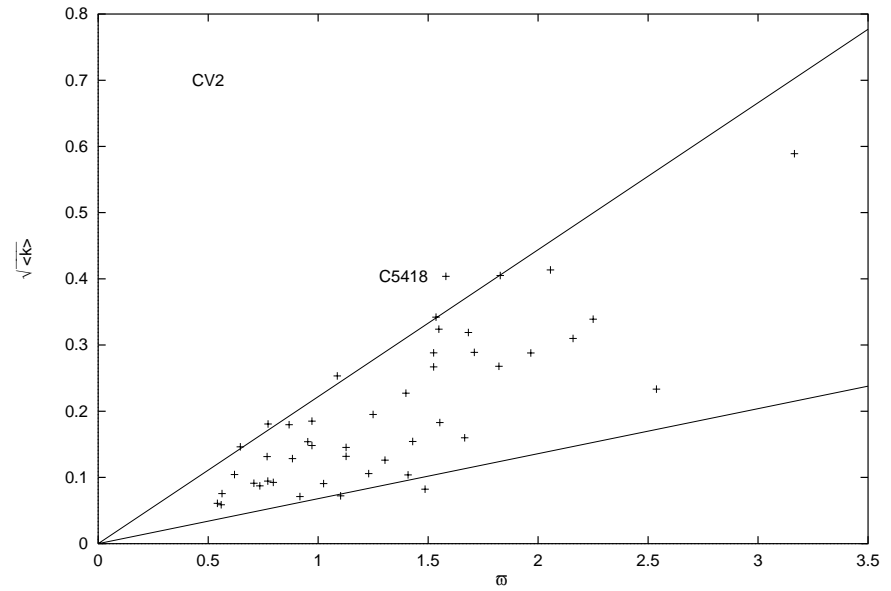

Fig. 2. The photometric relative angular radii $\langle k\rangle^{1 / 2}$ as a function of true parallaxes $\varpi$ in mas, for the CV2-group.

The latter quantity is a linear and homogeneous function of $\varpi$. Parallax errors predominate over photometric errors on $\langle k\rangle^{1 / 2}$. Within a restricted sample, say in a given photometric group, the distribution of true parallaxes may be assumed Gaussian, which is also the case of the quantity in Eq. (5). The $C_{\mathrm{R}^{-}}$ coefficient can then be averaged without being affected by the bias described by Smith \& Eichhorn (1996). This is the angular radius (in mas) shown by a star of photometric coefficient $\langle k\rangle$ and parallax $\varpi$ if its magnitude would have been $[1.08]_{0}=0$ and its radius have been $R=R_{\odot}$. In practice, the third member of Eq. (5) will be used to evaluate $C_{R}$, to be averaged for a given group (or class) of stars. The mean values $\left\langle R / R_{\odot}\right\rangle$ are then evaluated from $\Phi_{0}$, a quantity that correlates with groups and effective temperatures, and not with $\langle k\rangle^{1 / 2}$ (Paper I).

Individual estimates of $R / R_{\odot}$ may be derived from individual $\Phi_{0}$ 's as quoted in Paper I or from $\Phi_{0}\left(T_{\text {eff }}\right)$ relations, to be replaced in Eq. (5). They should not be averaged since the corresponding means could be biased.

\subsection{The $\langle k\rangle^{1 / 2}$ vs. $\varpi$ diagrams}

The above Eq. (5) can be written as

$$
\langle k\rangle^{1 / 2} \simeq \alpha \varpi
$$

where $\alpha \simeq\left(214.94 C_{R}\right)^{-1}$. That coefficient is expected to span a range delineated by those in $R$ and $\Phi_{0}$ for the considered group. The corresponding diagram of $\langle k\rangle^{1 / 2}$ vs. $\varpi$ should exhibit a populated interior of an angle with vertex at origin. This is the result shown by Knapik et al. (1998) for 34 CV2-stars (their Fig. 3), whereas the diagram with observed parallaxes $\varpi_{0}$ as abscissae did not have this property. We present here an updated version of the former diagram including $43 \mathrm{CV} 2$-stars and 46 SEDs (Fig. 2) that confirms the previous one. The diagrams for the groups $\mathrm{HC} 0+\mathrm{HC} 1, \mathrm{HC} 2$ to $\mathrm{HC} 5, \mathrm{CV} 1, \mathrm{CV} 3$ and CV4 are displayed in Fig. 3, and CV5 and CV6+CV7 in Fig. 4. The poorly-documented SCV-group is not shown.
Table 1. Minimum and maximum slopes estimated as described in Sect. 3.2. A strong increase is noticed from $\mathrm{HC} 3$ to $\mathrm{CV} 3$ followed by a flat portion (CV3-CV6). It illustrates the increase of photospheric radii along the sequence of groups.

\begin{tabular}{ccc}
\hline \hline $\mathrm{G}$ & $(\alpha \pm \Delta \alpha)_{\min }$ & $(\alpha \pm \Delta \alpha)_{\max }$ \\
\hline $\mathrm{HC} 1$ & $(1.48 \pm 0.10) \times 10^{-2}$ & $(3.80 \pm 0.20) \times 10^{-2}$ \\
$\mathrm{HC} 2$ & $(1.95 \pm 0.13) \times 10^{-2}$ & $(4.48 \pm 0.15) \times 10^{-2}$ \\
$\mathrm{HC} 3$ & $(2.27 \pm 0.15) \times 10^{-2}$ & $(7.84 \pm 0.40) \times 10^{-2}$ \\
$\mathrm{HC} 4$ & $(4.36 \pm 0.63) \times 10^{-2}$ & $(1.18 \pm 0.18) \times 10^{-1}$ \\
$\mathrm{HC} 5$ & $(6.41 \pm 0.51) \times 10^{-2}$ & $(1.55 \pm 0.28) \times 10^{-1}$ \\
$\mathrm{CV} 1$ & $(4.93 \pm 0.85) \times 10^{-2}$ & $(1.87 \pm 0.13) \times 10^{-1}$ \\
$\mathrm{CV} 2$ & $(6.81 \pm 0.98) \times 10^{-2}$ & $(2.22 \pm 0.11) \times 10^{-1}$ \\
$\mathrm{CV} 3$ & $(8.43 \pm 1.22) \times 10^{-2}$ & $(2.04 \pm 0.16) \times 10^{-1}$ \\
$\mathrm{CV} 4$ & $(1.16 \pm 0.12) \times 10^{-1}$ & $(2.47 \pm 0.24) \times 10^{-1}$ \\
CV5 & $(9.41 \pm 1.17) \times 10^{-2}$ & $(2.27 \pm 0.20) \times 10^{-1}$ \\
$\mathrm{CV} 6$ & $(1.10 \pm 0.15) \times 10^{-1}$ & $(2.44 \pm 0.30) \times 10^{-1}$ \\
\hline
\end{tabular}

Unfortunately, the number of stars per diagram is rather limited. The group CV2 was studied in detail. The Gaussian distribution

$N(\alpha) \mathrm{d} \alpha=\frac{N}{(2 \pi)^{1 / 2} \sigma} \exp -\frac{(\alpha-\langle\alpha\rangle)^{2}}{2 \sigma^{2}} \mathrm{~d} \alpha$

could be fit around the arithmetic mean

$\langle\alpha\rangle \simeq 0.148 \pm 0.052$.

Despite a large dispersion, a satisfactory solution was found with $N \simeq 0.859$ and $\sigma \simeq 0.057$, that is

$N(\alpha) \mathrm{d} \alpha \simeq 6.050 \exp \left[-156(\alpha-0.148)^{2}\right] \mathrm{d} \alpha$.

Two linear relations shown as full lines in Fig. 2 were derived which correspond roughly to

$\alpha_{\min } \simeq\langle\alpha\rangle-1.36 \sigma$

and

$\alpha_{\max } \simeq\langle\alpha\rangle+1.36 \sigma$

which results in $83 \%$ of data falling inside the materialized sector $\left(\alpha_{\min }, \alpha_{\max }\right)$. There is no significant departure except perhaps for C5418 = V460 Cyg.

Fits to the other diagrams for $\mathrm{HC}$ and $\mathrm{CV}$-groups were also derived, which proved frequently more difficult to obtain. The obtained estimates are quoted in Table 1 for the sequence of groups in our classification. They roughly correspond to inferior and superior limits in relative radii $R / R_{\odot}$, for a given range in $\Phi_{0}$. A general increase of both limits is noticed along the sequence of photometric groups which is moderate till HC3 and then from CV3 to CV6, where steady extremas at nearly $0.10 \pm 0.01$ and $0.23 \pm 0.02$ are reached. Such flattenings at both ends of the sequence will be found again while deriving luminosities and studying the locus of carbon giants in the HR diagram (Sects. 5 to 7 ). 

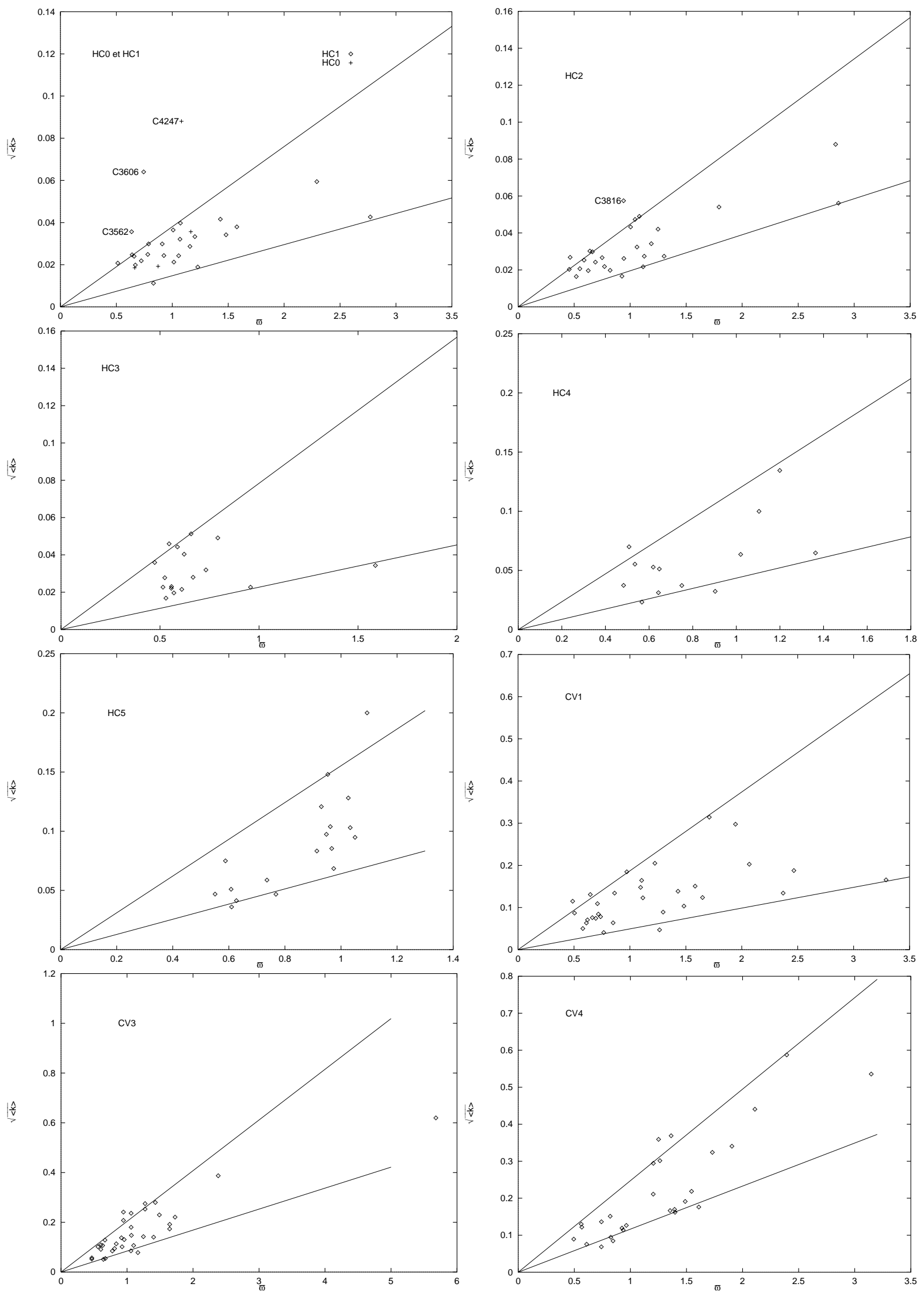

Fig. 3. Same diagram as Fig. 2 for the groups $\mathrm{HC} 0+\mathrm{HC} 1, \mathrm{HC} 2$ to $\mathrm{HC} 5$, and $\mathrm{CV} 1, \mathrm{CV} 3$ and $\mathrm{CV} 4$. 

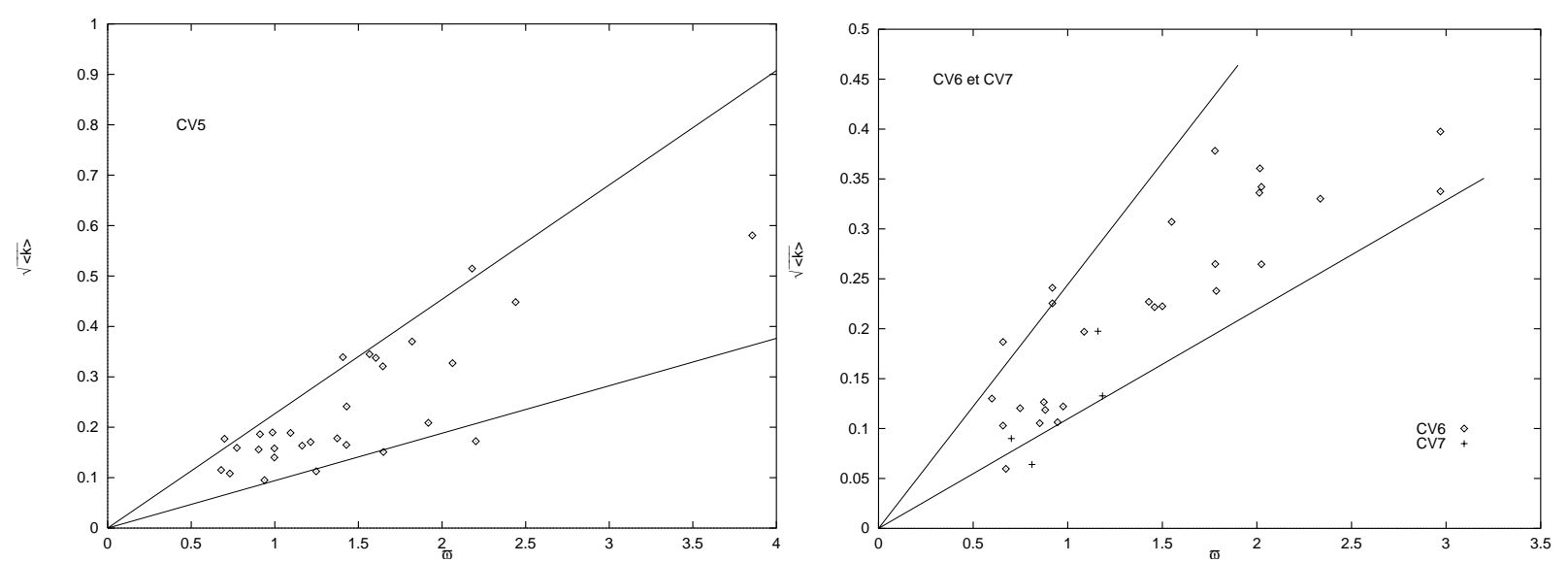

Fig. 4. Same diagram as Figs. 2 and 3 for the groups CV5 and CV6+CV7.

\subsection{The $C_{L}$-coefficient}

In order to again avoid the bias described by Smith \& Eichhorn (1996), we introduce now the coefficient

$C_{\mathrm{L}}=\left(L / L_{\odot}\right)^{-1 / 2}$

from luminosity in solar units. We used the coefficient

$C_{T_{\text {eff }}}=\left(\frac{\Phi_{0}}{2}\right)\left(\frac{T_{\text {eff }}}{T_{\text {eff } \odot}}\right)^{2}$

in Paper I (Eq. 36). Substituting Eq. (5), we obtain

$C_{\mathrm{L}}=C_{\mathrm{R}} / C_{T_{\mathrm{eff}}}$.

From Eq. (43) of Paper I, viz.

$C_{T_{\text {eff }}}=\frac{10^{-0.2\left(m_{\text {bol }}-M_{\text {bol }}\right)+2}}{214.94\langle k\rangle^{1 / 2}}$

and the above Eq. (5), we finally transform Eq. (12) into

$C_{\mathrm{L}}=\frac{\varpi_{\mathrm{mas}}}{10^{-0.2\left(m_{\mathrm{bol}}-M_{\mathrm{bol} \odot}\right)+2}}$

This latter relation shows that $C_{\mathrm{L}}$, like $C_{\mathrm{R}}$, is a linear and homogeneous function of the true parallax $\varpi$. Thus it can be averaged without being affected by the above-mentioned bias. The coefficient can be calculated from Eq. (16) with Eq. (14) used as a checking relation. We adopted $M_{\text {bol } \odot} \simeq 4.73$. The coefficients thus obtained can be averaged and estimates of mean luminosities derived from Eq. (12) for a group or class of carbon giants. Finally we adopt mean bolometric luminosities as obtained from

$\left\langle M_{\mathrm{bol}}\right\rangle-M_{\mathrm{bol} \odot} \simeq-2.5 \log \left\langle L / L_{\odot}\right\rangle \cdot$

Individual values of $L / L_{\odot}$ can be derived from Eqs. (16) and (12) i.e. from individual values of the $C_{\mathrm{L}}$ coefficients. They should not be averaged due to the mentioned bias. The same statement applies to individual absolute bolometric magnitudes. We shall use the latter to locate every carbon star in the HR diagram (see Sect. 7).

\subsection{The data for HIPPARCOS carbon stars}

The values of $C_{\mathrm{R}}$ and $C_{\mathrm{L}}$ were calculated for about 300 HIPPARCOS carbon stars with usable astrometric data. The corresponding values of $C_{T_{\text {eff }}}$ from Paper I were completed with the 81 additional stars of Sect. 2.2. Unfortunately, a few stars from initial proposals were missed. In addition to C3236 $=\mathrm{SS}$ Vir and C5496 = RX Peg, which were not correctly inserted in the entry catalogue (INCA), erroneous identifications affected C1616 = BK CMi, C1787 = BE CMi, C3614 = NSV7110, C4241 = U Lyr, C5371 = LU Cep and C5970 = V532 Cas. In addition HIC 22767 is not C808 = IRAS 04505+2241. No astrometric solution is given for C5721 = V363 Lac, and SU Tau $=$ HD247925, a RCB variable, was replaced by HD247901.

The values of $C_{\mathrm{R}}$ were calculated from Eq. (5), making use of true parallaxes $\varpi$ derived from HIPPARCOS observations, and the mean relative angular diameter $\langle k\rangle^{1 / 2}$ from multicolor photometry (see Sect. 3). The parallaxes were then used in Eq. (16) together with apparent bolometric magnitudes $m_{\text {bol }}$ from Paper I, assuming $M_{\text {bol } \odot} \simeq 4.73$ (see Sect. 5). Individual radii and luminosities were also derived as described at the ends of Sects. 3.1 and 3.3. The deduced individual absolute bolometric magnitudes are quoted in Table 2 , which is only available at CDS. This table is a modified, augmented and updated version of Table 10 of Paper I available at CDS. It contains 508 entries corresponding to 508 analyzed SEDs. Effective temperatures, interstellar (and eventually circumstellar) extinctions, classification in our photometric groups, bolometric magnitudes and comments are quoted there. For about 370 objects, absolute bolometric magnitudes are given as derived from true parallaxes.

\section{Mean data in terms of photometric groups}

Mean photospheric radii and mean luminosities or equivalently mean absolute bolometric magnitudes, are summarized and briefly discussed.

\subsection{The carbon stars}

Mean values and dispersions of the three coefficients $C_{T_{\mathrm{eff}}}, C_{\mathrm{R}}$ and $C_{\mathrm{L}}$, are quoted in Table 3 , with normalization factors of 
1,10 and 100 respectively. Then mean luminosities $\left(L / L_{\odot}\right)$ are deduced from Eq. (12) and mean radii $\left\langle R / R_{\odot}\right\rangle$ from Eq. (5). Finally mean absolute bolometric magnitudes were calculated from Eq. (17). The corresponding ranges in radii and absolute bolometric magnitudes are denoted as (inf;sup) in Cols. 8 and 9. Following our approach (Sects. 3.1 and 3.3), these mean values are in principle free of the bias described by Smith \& Eichhorn (1996). They are however affected by the Malmquist bias whose influence was studied in Knapik et al. (1998) and in Sect. 2 of Paper II. Here, the corrections for the effect of the Malmquist bias on bolometric absolute magnitudes are applied as estimated in Sect. 4 of Paper II. The values (Sect. 2.3) are small when compared to dispersions in Table 3.

After steady values close to $(6.5 \pm 0.4) \times 10^{-2}$ observed for the groups $\mathrm{HC} 0$ to $\mathrm{HC} 2, C_{L}$ continuously decreases along the sequence HC3-HC5 and then along CV1-CV7. Since the averaged effective temperatures diminish continuously along that sequence, this is a consequence of $\left\langle L / L_{\odot}\right\rangle$ increasing with decreasing $\left\langle T_{\text {eff }}\right\rangle$ (see Sect. 7 and the HR diagram in Figs. 8 and $9)$. This is in good agreement with the evolutionary tracks as predicted for stars of low and intermediate masses. At the same time, the $C_{\mathrm{R}}$-values decrease and $\left\langle R / R_{\odot}\right\rangle$ increases.

It can be seen that the whole domain of the brightest normal giants (luminosity class III), bright giants (II) and finally faint supergiants (Ib), is populated, with no gap left, from $\left\langle M_{\text {bol }}\right\rangle \simeq-1.1$ to -5.8 . The BaII stars, which are fainter on average (Sect. 4.2), are observed down to $\left\langle M_{\text {bol }}\right\rangle \simeq 1.0$. A peculiar case is represented by the SCV-group. Knapik et al. (1999) found it to stand as a transition between $\mathrm{S}$ and $\mathrm{CV}$-stars. We adopted the $\Phi_{0}$-value of group CV4 since it leads to $\left\langle T_{\text {eff }}\right\rangle$ close to $2775 \mathrm{~K}$, the mean value of the CV4-group. The SCV-group does exhibit a luminosity of $16000 L_{\odot}$ that is $\left\langle M_{\text {bol }}\right\rangle \simeq-5.8$, close to the values for CV7. Both groups are however poorlydocumented. For instance, including the underluminous CV7star C2334 $(n=16)$ leads to $\left\langle M_{\mathrm{bol}}\right\rangle \simeq-5.4$ instead of -5.8 (Table 3). At the faint end, the three groups $\mathrm{HC} 0, \mathrm{HC} 1$ and HC2 directly yield $\left\langle M_{\text {bol }}\right\rangle \simeq(-1.2 \pm 0.13)$ i.e. about $\left(240 L_{\odot}\right)$ with uncorrected values, and $\left\langle M_{\text {bol }}\right\rangle \simeq(-1.0 \pm 0.2)$ when our correction for the Malmquist bias is applied. Their locus is found at the junction between classes II and IIIa, well above the clump of classes IIIb-IIIab. A practically continuous luminosity distribution is observed for carbon giants in Table 3, and delineating discrete luminosity classes is hardly justified. This is also clear for HIPPARCOS oxygen-rich giants in the HR diagrams published by Perryman et al. (1995). From mean absolute magnitudes of Table 3 and bolometric corrections of Sect. 2, and mean color indices of Table 11 in Paper I, we found $\left\langle M_{K}\right\rangle \simeq-2.5$ to -3.3 for early HC-stars.

Cooler R-stars, e.g. HC3, are still brighter with $\left\langle M_{K}\right\rangle \simeq$ -4.4 . Since HCO stars are very few, the obtained mean value $\left\langle M_{K}\right\rangle \simeq-3.0$ is dominated by $\mathrm{HC} 1$ and $\mathrm{HC} 2$ stars. This is 1 mag brighter than found by Knapp et al. (2001) for corresponding R0-R3 stars. They adopted a Gaussian distribution of $\left\langle M_{K}\right\rangle \simeq-2.0$ and standard deviation $1.0 \mathrm{mag}$, very close to the $M_{K} \simeq-1.6$ value for clump red giants of Alves (2000). We actually found this is the case for many BaII stars that are on average fainter than early $\mathrm{R}$ stars (Sect. 4.2).
Our method (Knapik et al. 1998, Paper II) and that of Knapp et al. (2001) are statistical in nature (see also Pourbaix \& Jorissen 2000), but they differ in several aspects. The samples used (HC1 to HC3-stars, or equivalently spectral types R0 to R3) include nearly the same HIPPARCOS stars. Pourbaix \& Jorissen however re-analyzed the HIPPARCOS Intermediate Astrometric Data, while the published observed parallaxes and proper motions (ESA 1997) were used in our work. The biases as described in Sect. 2.3 are not accounted for in the same way. An additional difference is that Knapp et al. (2001) assumed that the absolute magnitudes are distributed about a single mean absolute magnitude in the infrared $\left(\left\langle M_{K}\right\rangle \simeq-2\right)$ with a 1 mag intrinsic standard deviation, as consistent with their analysis. On the contrary, we find a gradient on $\left\langle M_{K}\right\rangle$ from HC1 to HC3, as mentioned above. Knapp et al. (2001) mention a possible shift of their results, but toward $\left\langle M_{K}\right\rangle \simeq-1$. We have simulated possible systematic effects on our mean absolute magnitudes. Varying the $q$-slope of Sect. 2.3 from 2.35 to 3 , up to $0.3 \mathrm{mag}$ shifts are deduced (possible underestimates of the Lutz-Kelker bias). A value in excess of 4 would be required to reach a 1 mag-shift, which we exclude. The corrections for the Malmquist bias are known to within 0.1-0.15 mag. We consider the combined effect of both uncertainties should not exceed 0.4 mag in the worst case.

\subsection{The Ball stars}

Bergeat \& Knapik (1997) studied a sample of 52 BaII stars they locate in the observational HR diagram, i.e. absolute visual magnitudes vs. spectral types, or almost equivalently oxygen-rich photometric groups. HIPPARCOS data of a much larger sample were used, together with radial velocities, by Mennessier et al. (1997). Both studies conclude this spectroscopic group is an inhomogeneous one, spreading upon luminosity classes V to Ib. Preparing the theoretical HR diagram $\left(M_{\mathrm{bol}}, T_{\mathrm{eff}}\right)$ of Sect. 7 , we have integrated the 44 bestdocumented dereddened SEDs and derived the values of the apparent bolometric magnitudes $m_{\text {bol }}$. For 29 additional stars, mean bolometric corrections

$\left\langle B C_{V}\right\rangle=\left\langle m_{\mathrm{bol}}-V_{0}\right\rangle=\left\langle M_{\mathrm{bol}}-M_{V}\right\rangle$

were used. The reader is referred to Bergeat \& Knapik (1997) for the use of observed parallaxes. We mention here that HD199394 is classified as IIIb instead of IIIa as was erroneously quoted in their Table 1 . In the present study, we exclude the supergiants (class Ib or photometric groups sg) and the dwarfs above the main sequence and subgiants (classes $\mathrm{V}$ to IV or photometric groups $\mathrm{d}$ and $\mathrm{g}$ ), and the dwarfs on the main sequence (class V or groups d). We only keep the BaII giants either bright (class II) or normal (class III subdivided into IIIa, IIIab and IIIb, or photometric group g). Revised (postHIPPARCOS) definitions of classes III can be found in Keenan \& Barnbaum (1999). For the corresponding 56 stars, we obtained

$\left\langle M_{\mathrm{bol}}\right\rangle \simeq-0.27 \pm 1.26$ 
Table 3. Mean data obtained for the fourteen photometric groups of carbon stars (HC, CV and transition SCV: Col. 1). The mean effective temperatures and reference relative angular diameters in mas from Paper I are quoted in Cols. 2 and 3 respectively, as well as the corresponding mean coefficients in Col. 4: $c_{T_{\text {eff }}} \pm \Delta c_{T_{\text {eff }}}=\left\langle C_{T_{\text {eff }}} \pm \Delta C_{T_{\text {eff }}}\right\rangle$. The reduced mean coefficients $c_{\mathrm{R}} \pm \Delta c_{\mathrm{R}}=10\left\langle C_{\mathrm{R}} \pm \Delta C_{\mathrm{R}}\right\rangle$ and $c_{\mathrm{L}} \pm \Delta c_{\mathrm{L}}=$ $10^{2}\left\langle C_{\mathrm{L}} \pm \Delta C_{\mathrm{L}}\right\rangle$ are given in Cols. 5 and 6 respectively. The mean luminosities and radii in solar units, are shown in Cols. 7 and 9 together with the ranges (inf; sup) of the latter. The deduced mean absolute bolometric magnitudes and corresponding ranges can be found in Col. 8. Mean effective temperatures are quoted in Col. 10, which are very close to those of Paper I (Col. 2); ${ }^{\text {a }}$ without C4247 brighter than the other 3 stars; ${ }^{\text {b }}$ without the underluminous C2334; ${ }^{\mathrm{c}}$ values corrected for the Malmquist bias (Paper II).

\begin{tabular}{|c|c|c|c|c|c|c|c|c|c|}
\hline G & eff $>n$ & $\left\langle\Phi_{0}\right\rangle$ & $T_{\text {eff }} \pm \Delta c_{T_{\text {eff }}}$ & $\pm \Delta c_{R}$ & $\pm \Delta c_{\mathrm{L}}$ & $\left\langle L / L_{\odot}\right\rangle$ & $\left\langle M_{\mathrm{bol}}\right.$ & $\left\langle R / R_{\odot}\right\rangle$ & $\left\langle T_{\text {eff }}\right\rangle$ \\
\hline $\mathrm{HCO}$ & & 5.94 & $843 \pm 0$. & $7 \pm 0.65$ & $5 \pm 2.28$ & 38 & & & 5645 \\
\hline $\mathrm{HCO}^{(a)}$ & & 5.94 & $2.843 \pm 0.145$ & $1.77 \pm 0.31$ & $6.17 \pm 1.24$ & 38 & $;-1.81$ & 0 & 5675 \\
\hline $\mathrm{HC} 1$ & 4890 & 6.72 & $2.416 \pm 0$ & $1.46 \pm 0.38$ & $6.96 \pm 2.68$ & 2 & $.06-0.35 ;-2.11$ & & 4895 \\
\hline $\mathrm{HC} 1^{(c)}$ & & & & $2.0 \pm 0.64$ & \pm 3.10 & 1 & $-0.740 .0 ;-1.8$ & 5 & \\
\hline $\mathrm{HC} 2$ & 4290 & 8.38 & $2.305 \pm 0.1$ & $1.48 \pm 0.51$ & $6.44 \pm 2.24$ & 240 & $-1.22-0$ & & 4280 \\
\hline $\mathrm{HC} 2^{(c)}$ & & & & 1.6 & $7.09 \pm 2.5$ & 2 & $-1.02-0.3 ;-2$ & 9 & \\
\hline $\mathrm{HC} 3$ & 400519 & 9.41 & $2.256 \pm 0.056$ & $1.09 \pm 0.44$ & $4.54 \pm 2.16$ & 48 & $-1.99-1.15 ;-3.36$ & & 3995 \\
\hline $\mathrm{HC} 4$ & 515 & .57 & 2.275 & 0.65 & 5 & 1 & $-3.00-$ & U & 3980 \\
\hline $\mathrm{HC} 5$ & 348020 & 11.9 & $2.151 \pm 0.093$ & $0.517 \pm 0.153$ & $2.40 \pm 0.70$ & 1735 & $-3.37-2.81 ;-4.12$ & ;164 & 3465 \\
\hline $\mathrm{CV} 1$ & 3285 & 13.6 & $2.197 \pm 0.069$ & $0.438 \pm 0.171$ & $2.04 \pm 0.79$ & 2400 & $-3.72-3.01 ;-4.79$ & 256 & 3275 \\
\hline $\mathrm{CV} 1^{(c)}$ & & & & $0.48 \pm 0.18$ & $2.14 \pm 0.87$ & 2190 & $-3.62-3.00 ;-4.70$ & 142 & \\
\hline $\mathrm{CV} 2$ & 303 & 16.5 & $2.293 \pm$ & $0.359 \pm 0.149$ & $1.53 \pm 0.57$ & 4255 & $-4.34-3.65 ;-5.35$ & 230 & 3040 \\
\hline $\mathrm{CV} 2^{(c)}$ & & & & $0.38 \pm 0.17$ & $1.61 \pm 0.63$ & 3870 & $-4.24-3.50 ;-5.30$ & 216 & \\
\hline $\mathrm{CV} 3$ & 291543 & 18.2 & $2.337 \pm 0.047$ & $0.349 \pm 0.119$ & $1.50 \pm 0.52$ & 4450 & $-4.39-3.74 ;-5.32$ & $261195 ; 395$ & 2920 \\
\hline $\mathrm{CV}^{(c)}$ & & & & $0.36 \pm 0.12$ & $1.57 \pm 0.57$ & 4055 & $-4.29-3.70 ;-5.20$ & 253 & \\
\hline $\mathrm{CV} 4$ & 277532 & 20.5 & $2.370 \pm 0.033$ & $0.305 \pm 0.129$ & $1.32 \pm 0.54$ & 5700 & $-4.66-3.91 ;-5.80$ & $336236 ; 583$ & 2770 \\
\hline $\mathrm{CV} 4^{(c)}$ & & & & $0.33 \pm 0.13$ & $1.39 \pm 0.59$ & 5200 & $-4.56-3.80 ;-5.70$ & $313220 ; 543$ & \\
\hline CV5 & 264544 & 23.5 & $2.434 \pm 0.045$ & $0.299 \pm 0.092$ & $1.23 \pm 0.37$ & 659 & $-4.82-4.24 ;-5.60$ & 392299 & 2630 \\
\hline $\mathrm{CV} 5^{(c)}$ & & & & $0.30 \pm 0.11$ & $1.29 \pm 0.41$ & 6030 & $-4.72-4.20 ;-5.50$ & $394299 ; 567$ & \\
\hline CV6 & 244549 & 29.4 & $2.610 \pm 0.061$ & $0.307 \pm 0.078$ & $1.11 \pm 0.32$ & 8130 & $-5.05-4.49 ;-5.79$ & $2 ; 644$ & 2430 \\
\hline $\mathrm{CV}^{(c)}$ & & & & $0.31 \pm 0.08$ & $1.16 \pm 0.35$ & 7450 & $-4.95-4.40 ;-5.70$ & $467372 ; 628$ & \\
\hline CV7 & 195516 & 69.3 & $3.932 \pm 0.506$ & $0.358 \pm 0.14$ & $0.93 \pm 0.40$ & 11580 & $-5.43-4.6 ;-6.8$ & $969700 ; 1580$ & 1945 \\
\hline $\mathrm{CV}^{(b)}$ & & 69.3 & $3.932 \pm 0.506$ & $0.311 \pm 0.09$ & $0.78 \pm 0.27$ & 16400 & $-5.81-5.2 ;-6.7$ & $1112870 ; 1560$ & 1945 \\
\hline $\mathrm{SCV}$ & 775: 11 & 20.5 & $2.395 \pm 0.080$ & $0.188 \pm 0.028$ & $0.79 \pm 0.09$ & 15835 & $-5.77-5.51 ;-6.06$ & $545475 ; 641$ & 2790 \\
\hline
\end{tabular}

Table 4. Mean data obtained for the five variability classes of carbon stars: M(1) denotes the full sample of carbon Miras, whereas the underluminous Miras T Lyn and RZ Peg were removed from the M(2)-sample. The meaning of symbols is the same as in Table 3, except for the "equivalent" group $\langle\mathrm{G}\rangle$ (see text for details).

\begin{tabular}{|c|c|c|c|c|c|c|c|c|c|c|c|}
\hline $\mathrm{Cl}$ & $c_{\mathrm{R}} \pm \Delta c_{\mathrm{R}}$ & $c_{\mathrm{L}} \pm \Delta c_{\mathrm{L}}$ & $\left\langle L / L_{\odot}\right\rangle$ & $\left\langle M_{\mathrm{bol}}\right\rangle$ & (inf;sup) & $\left\langle T_{\text {eff }}\right\rangle$ & $\left\langle\Phi_{0}\right\rangle$ & $\left\langle R_{\mathrm{p}} / R_{\odot}\right\rangle$ & (inf;sup) & $\langle\mathrm{G}\rangle$ & $n$ \\
\hline Cst & $1.40 \pm 0.58$ & $6.02 \pm 2.37$ & 275 & -1.37 & $(-0.65 ;-2.46)$ & $4375 \pm 475$ & 8.15 & 30 & $(20 ; 50)$ & $\mathrm{HC} 2$ & 73 \\
\hline $\mathrm{Lb}$ & $0.409 \pm 0.179$ & $1.80 \pm 0.86$ & 3075 & -3.99 & $(-3.14 ;-5.39)$ & $2950 \pm 330$ & 18.2 & 225 & $(155 ; 400)$ & CV3 & 111 \\
\hline $\mathrm{SRb}$ & $0.337 \pm 0.147$ & $1.50 \pm 0.65$ & 4475 & -4.40 & $(-3.61 ;-5.64)$ & $2895 \pm 325$ & 18.2 & 270 & $(190 ; 480)$ & $\mathrm{CV} 3+$ & 60 \\
\hline SRa & $0.315 \pm 0.130$ & $1.31 \pm 0.58$ & 5860 & -4.69 & $(-3.90 ;-5.96)$ & $2730 \pm 270$ & 20.5 & 325 & $(230 ; 555)$ & CV4 & 20 \\
\hline $\mathrm{M}(1)$ & $0.332 \pm 0.117$ & $1.30 \pm 0.57$ & 5965 & -4.71 & $(-3.92 ;-5.97)$ & $2550 \pm 540$ & 26.5 & 400 & $(295 ; 615)$ & CV5-6 & 53 \\
\hline $\mathrm{M}(2)$ & $0.312 \pm 0.081$ & $1.19 \pm 0.40$ & 7015 & -4.89 & $(-4.26 ;-5.77)$ & $2535 \pm 540$ & 26.5 & 425 & $(340 ; 570)$ & CV5-6 & 51 \\
\hline
\end{tabular}




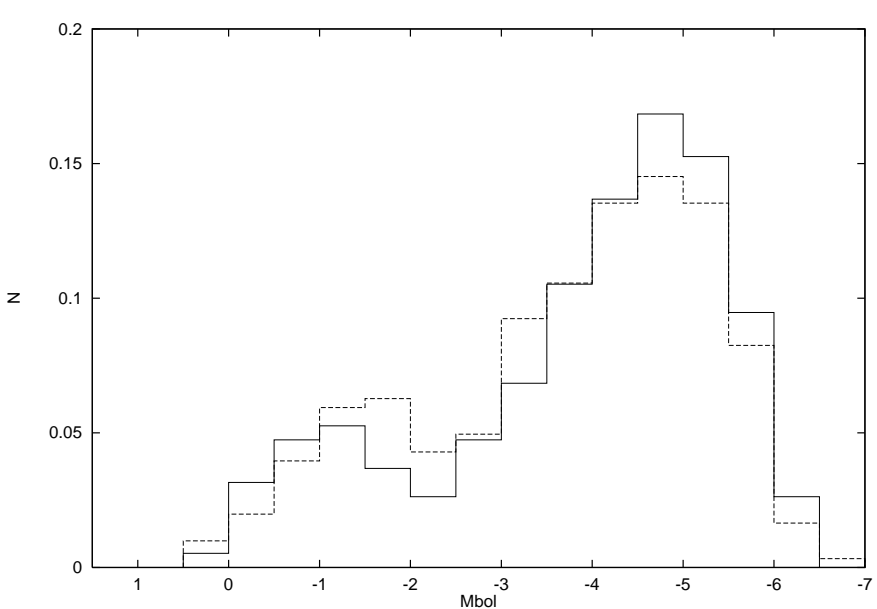

Fig. 5. The luminosity function (LF) of Galactic carbon giants in the Sun vicinity (dashed lines). The LF for stars with $\varpi \geq 0.9$ mas presumably free from effects of the Malmquist bias is shown with full lines ("MW" hereafter).

This is fainter than the faintest value deduced for $\mathrm{HC}$ carbon stars (about -1 ), even if both samples substantially overlap. Apart from a concentration around revised class IIIb (the "clump"), no marked structure is observed.

The effective temperatures were derived from Table B.7 of Knapik (1999), a calibration of mean values for photometric groups adapted from Perrin et al. (1998) and Richichi et al. (1999). Individual values of $M_{\mathrm{bol}}$ and $T_{\mathrm{eff}}$ can be found at the end of Table 2 for 62 BaII stars and $T_{\text {eff }}$ for 5 additional stars.

\section{Mean data in terms of variability classes}

According to the classification of Kholopov et al. (1985), we consider a sequence of increasing variability, namely "Cst, Lb, $\mathrm{SRb}, \mathrm{SRa}$ and M" from constant stars to Miras including irregular variables (Lb) and semi-regulars with either small amplitude of variations ( $\mathrm{SRb}$ ) or large amplitude (SRa). We used the same classification in Paper II, in the study of the reflex solar velocity in terms of variability classes. The approach used here is similar to that of Sect. 4, except for $\left\langle T_{\text {eff }}\right\rangle$ which is a "mean" value deduced from Eq. (13) where $\left\langle C_{T_{\text {eff }}}\right\rangle$ itself is derived from $\left\langle C_{\mathrm{R}}\right\rangle$ and $\left\langle C_{\mathrm{L}}\right\rangle$ replaced into Eq. (14) which is assumed to hold for mean values. The relation $\left\langle\Phi_{0}\left(T_{\text {eff }}\right)\right\rangle$ of Paper I was adopted. The corresponding $\left\langle\Phi_{0}\right\rangle$ allows the determination of an "equivalent" group $\langle\mathrm{G}\rangle$ for each variability class. The results are given in Table 4.

The luminosity and the photospheric radius increase and the effective temperature decreases, on average, while following the sequence of increasing variability: Cst, Lb, SRb, SRa and $M$. The stars classified as constant (i.e. for which no photometric variations larger than a few hundredths of a magnitude could be evidenced) are essentially early HC-giants from $\mathrm{HC} 0$ to $\mathrm{HC} 3$, many $\mathrm{HC} 4$ and $\mathrm{HC} 5$-stars being variables. We obtained $\mathrm{HC} 2$ as their equivalent group with good consistency for mean luminosity, photospheric radius and effective temperature. Most variable carbon stars belong to a CV-group as initially intended and a strong discontinuity in properties appears when skipping from Cst to Lb. There is then a slow

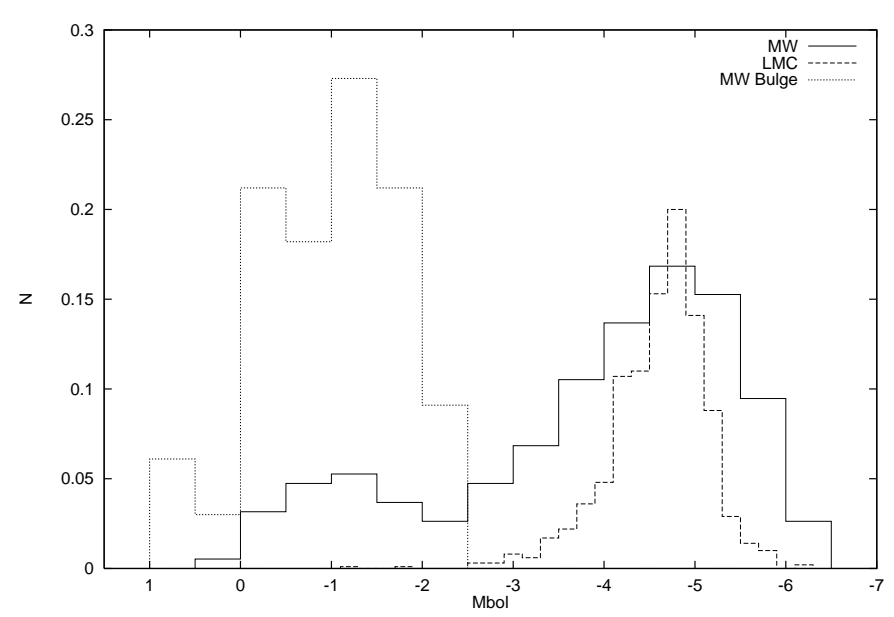

Fig. 6. The MW-LF of Fig. 5, confronted to the LF of the Galactic bulge (MWB, 33 stars; Rich 1989) and to the LF of the LMC (LMC, 895 stars; Costa \& Frogel 1996).

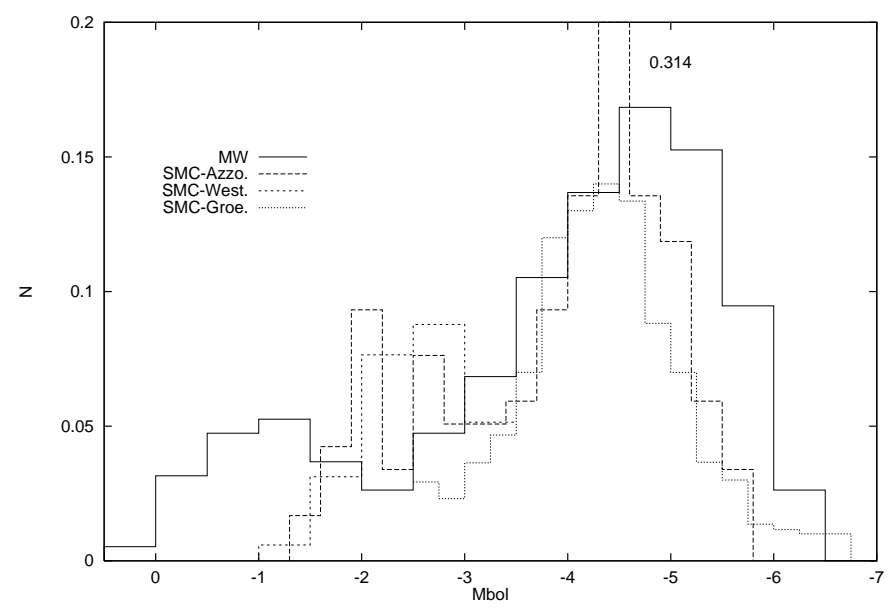

Fig. 7. The MW-LF of Fig. 5, confronted to the SMC-LFs of Azzopardi (1993; "SMC-Azzo."), Westerlund et al. (1995; "SMCWest.”), and Groenewegen (1998; "SMC-Groe.”).

evolution along the sequence from $\mathrm{Lb}$ to $\mathrm{M}$, with increasing mean luminosities and photospheric radii, and decreasing mean effective temperatures. Considering dispersions, the regular evolution along the sequence is more convincing than the differences found between two successive classes. The mean $\left\langle M_{\text {bol }}\right\rangle \simeq-4.7$, i.e. $\left\langle L / L_{\odot}\right\rangle \simeq 4900$, value obtained for the full sample M(1) of carbon Miras, is similar to that quoted for SRa variables. From HIPPARCOS data, a few Miras proved to be strongly underluminous (e.g. Bergeat et al. 1998): this is the case of C2165 = T Lyn and C5570=RZ Peg which we excluded from sample $\mathrm{M}(2)$. Then we obtained $\left\langle M_{\text {bol }}\right\rangle \simeq-4.9$, i.e. $\left\langle L / L_{\odot}\right\rangle \simeq 7100$. On average, the amplitudes of variations in carbon Miras are lower than those of their oxygen-rich counterparts. There is no gap between the former and large-amplitude carbon semiregulars classified SRa. The results of Table 4 can be interpreted in terms of increasing variability, on average, along the evolutive tracks in the TP-AGB region (Sect. 7). 


\section{The luminosity function (LF) of Galactic carbon giants in the Sun vicinity}

We have split the interval $M_{\text {bol }}=[-7.0 ;+0.5]$ into 15 bins 0.5 mag wide each. The distribution thus obtained will be referenced as the luminosity function (LF) of Galactic carbon giants in the Sun vicinity. Normalized to a total of unity, it is shown with dashed lines in Fig. 5, (HC-, CV- and SCV-stars are included; BaII stars are not). It can be calibrated in terms of the total surface density in the Sun vicinity projected on the Plane, of $(75.7 \pm 3.7) \mathrm{kpc}^{-2}$ as estimated in Paper II. The results for $M_{\text {bol }} \leq-5.5$ are probably underestimated due to missing CV6CV7 and SCV-stars, since a $10 \%$ increase was tentatively included in the above-mentioned density.

Two maxima are noticed at about $M_{\text {bol }} \simeq-1.5$ and -4.75 . They correspond to early HC-stars (HC1-HC3) and CV-stars (CV4-CV5) respectively. We recall now that the $\mathrm{HC}$ and $\mathrm{CV}$ stars are mainly members of two distinct populations on the grounds of space distributions and kinematics, respectively the thick disk component and the old (thin) disk component (Paper II). The former sample is contaminated by a spheroidal contribution, namely the $\mathrm{CH}$ stars. It is thus clear that the LF of Fig. 5 results from the superimposition of two LFs. This LF may be affected by the Malmquist bias as described in Knapik et al. (1998) and Paper II. It was shown there that the sample of HIPPARCOS carbon giants is reasonably complete within a sphere of radius $1.1 \mathrm{kpc}$ and that the effect of the Malmquist bias is quite moderate on most photometric groups (Paper II, corrections on mean values, of a few tenths of magnitude at most, were applied in Table 3). In order to minimize the effect of this bias on the Galactic LF, we selected the stars with large true parallaxes ( $\varpi \geq 0.9$ mas) and plotted the new LF with full lines in Fig. 5. It looks much like the previous one shown with dashed lines. The $M_{\text {bol }} \simeq-4.75$ maximum is nevertheless accentuated and the minimum near $M_{\mathrm{bol}} \simeq-2.25$ is slightly more pronounced. The half-height width of the $\mathrm{CV}$ portion also drops from about 6 mag to $4.5 \mathrm{mag}$. The maximum of HC-stars appears shifted from $M_{\text {bol }} \simeq-1.75$ to -1.25 . Those modifications can be understood in terms of reducing simultaneously the effect of the Malmquist bias and the incidence of parallax errors (which are on average smaller in the $\varpi \geq 0.9$ mas sample).

This latter LF for Galactic carbon giants in the Sun vicinity hereafter referred to as MW for Milky Way, is compared to the LFs of the Galactic bulge (MWB, 33 stars; Rich 1989), and to that of the Large Magellanic Cloud (LMC, 895 field C stars; Costa \& Frogel 1996) in Fig. 6. The distance modulus 18.5 (Bergeat et al. 1998) is adopted. In Fig. 7, the MWLF is confronted to the LFs obtained for the Small Magellanic Cloud (SMC) by Azzopardi (1993), Westerlund et al. (1995), and from the data of Rebeirot et al. (1993) by Groenewegen (1998). Our MW-LF appears as larger than the LMC-LF. Errors on true parallaxes certainly contribute, while all LMC-stars are assumed at a common distance from Sun.

The most remarkable feature is the absence of LMC faint carbon stars $\left(M_{\mathrm{bol}} \geq-2.5\right)$, which are HC-stars in the Galactic disk. The latter thick disk population (Paper II) has no equivalent amongst the 895 field C stars of Costa \& Frogel
(1996), with the possible exception of two stars located in the $[-1.9 ;-1.7]$ and $[-1.3 ;-1.1]$ intervals respectively. The luminosity ranges of carbon stars in LMC clusters are still narrower (e.g. Costa \& Frogel 1996). Faint LMC carbon stars were searched for with no success until now $\left(M_{\mathrm{bol}} \leq-3.3\right.$, Azzopardi 1999b). The discovery of 43 hot $\mathrm{CH}$ stars in the outer halo of the LMC (Hartwick \& Cowley 1988) which are as bright as $-4 \geq M_{\text {bol }} \geq-6$, (Feast \& Whitelock 1992), is possibly part of the answer: the hot carbon stars are few in the LMC and most of them reach high luminosities. As shown in Fig. 6, the carbon stars in the Galactic bulge (MWB) populate the range $1 \geq M_{\text {bol }} \geq-2.5$, much similar to that of the early HC-stars in the thick disk ("MW" with $M_{\text {bol }} \geq-2.5$ ). Both maxima are located at $M_{\text {bol }} \simeq-1.25 \pm 0.25$. The populations of hot carbon stars in the thick disk and Galactic bulge look similar. Very bright carbon stars $\left(M_{\mathrm{bol}} \simeq-6.8\right)$ were found recently in the LMC by van Loon et al. $(1998,1999)$. They exhibit strong mass loss and thick circumstellar shells. Absent from Fig. 6, they are the counterpart of our brightest galactic CV7-objects.

Finally, the comparison to the LFs published for the SMC, is given in Fig. 7. The main maximum is located at $M_{\mathrm{bol}} \simeq$ $-4.4 \pm 0.25$, fainter than the $M_{\text {bol }} \simeq-4.8 \pm 0.25$ value obtained for both LMC and Milky Way. A secondary maximum is marginally seen in the SMC at $M_{\text {bol }} \simeq-2.4 \pm 0.4$ when averaging the three plotted LFs. It is flanked on its left by a rapid decrease. Carbon stars as faint as $M_{\text {bol }} \simeq-1.0$ are observed. It will be interesting to check for any effect of limiting apparent magnitude. According to Azzopardi (1999b), the faint-end of the carbon star LF in SMC, as inferred from 161 stars, is $M_{\text {bol }} \simeq-1.4$, assuming a distance modulus of 18.8. Azzopardi et al. (1999) found in the Fornax dwarf spheroidal galaxy, lowluminosity objects as faint as $M_{\text {bol }} \simeq-1.2$, assuming a dust free distance modulus of 21.0. Low-metallicity systems $(Z=0.004$ is usually adopted for SMC) seem to have in common the existence of low-luminosity carbon stars. It is known from stellar modeling that dredge-up is favored at low metallicity (Rossi et al. 1999) when compared to the solar $Z=0.02$ value (e.g. Straniero et al. 1997), so that the minimum mass at which a star mixes to the envelope C-rich materials decreases with increasing $Z$. Most of those stars seem however too faint to have reached this advanced phase of evolution.

\section{The carbon and Ball giants in the HR diagram}

\subsection{The HR diagram}

The coordinates in Fig. 8, i.e. absolute bolometric magnitudes and effective temperatures, can be found in Table 2 (available at CDS) for nearly 370 objects, including

- about 290 carbon stars (HC and CV-groups, i.e. from early $\mathrm{R}$ to late $\mathrm{N}$ stars),

- $56 \mathrm{Ba}$ II giant stars (some of them are also HC-stars),

- 15 RCB variables mostly classified in oxygen-rich groups (HdC stars are classified $\mathrm{HC}$ and found immediately on their right side), 


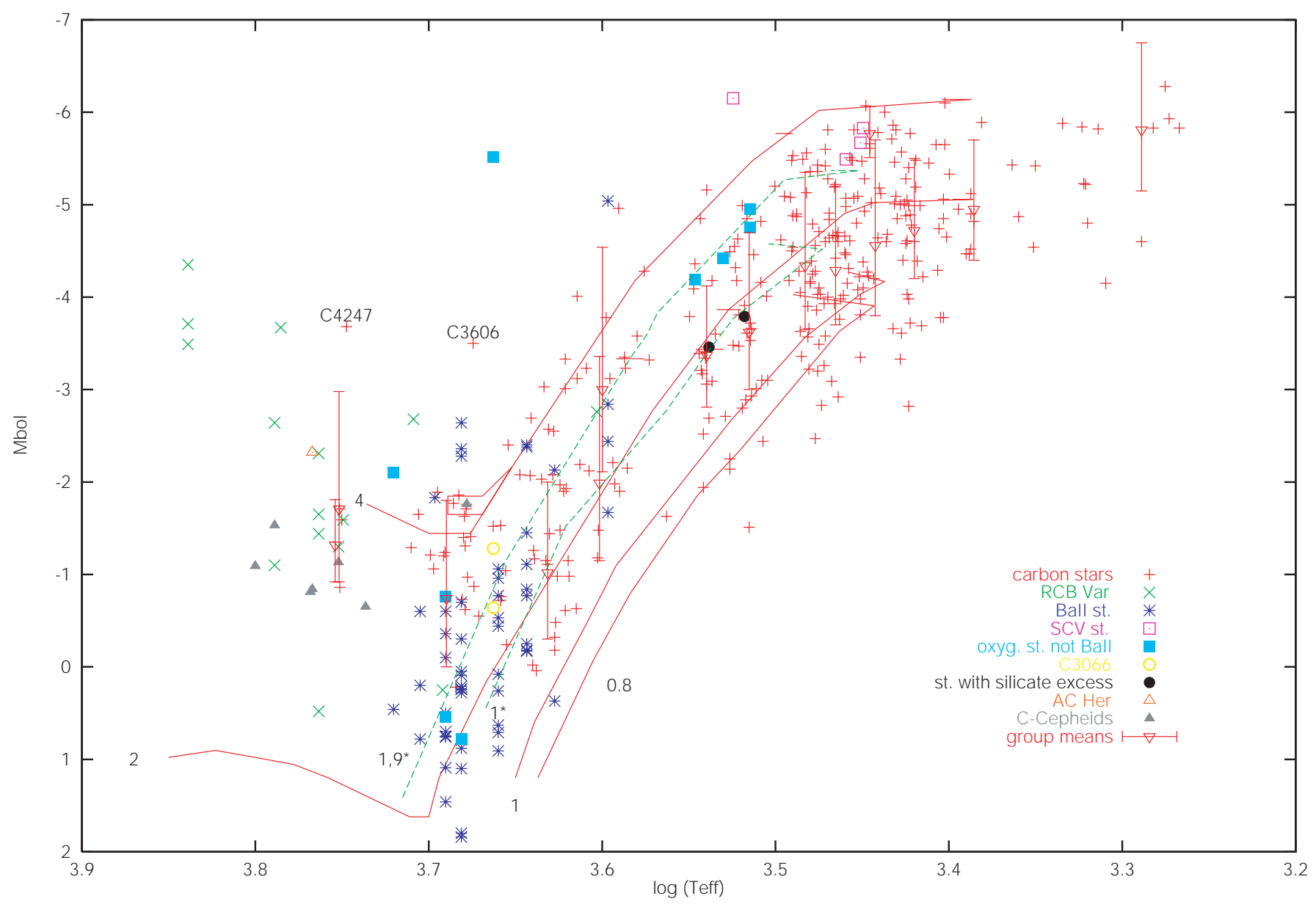

Fig. 8. The HR diagram of nearly 370 Galactic carbon and BaII giants, and related objects. Most stars populate a curved strip which correspond to theoretical evolutionary tracks of stars $(\mathrm{RGB}+\mathrm{AGB})$ with initial masses in the $0.8-4 M_{\odot}$ range. For $Z=0.02$, theoretical tracks were adapted from Bressan et al. (1993) and Marigo et al. (1996). For $Z=0.008$, tracks for 1 and $1.9 M_{\odot}$ labeled with asterisks were adapted from Fagotto et al. (1994c) and Marigo et al., which illustrate the resulting leftward shift. From left to right, mean values and dispersion bars are shown for the 14 carbon-rich photometric groups: $\mathrm{HC} 0$ (2 values: see text), $\mathrm{HC} 1 \mathrm{HC} 2, \mathrm{HC} 3$ and $\mathrm{HC} 4$ (at about the same $\log \left(T_{\text {eff }}\right) \simeq 3.6$ ), HC5, CV1, $\mathrm{CV} 2, \mathrm{CV} 3, \mathrm{SCV}$ and CV4 (at about the same $\log \left(T_{\text {eff }}\right) \simeq 3.445$ ), CV5, CV6 and CV7.

- 4 SCV-stars (a group which actually coincide with the SC spectroscopic type),

- 6 non-BaII stars, which finally proved to be oxygen-rich objects classified in the corresponding groups.

The derived luminosity data is essentially intended for statistical use, determining the loci of various star categories and also locating photometric groups. Departing from this rule, we also consider a few individual stars with remarkable features

- 2 carbon stars with silicate-type excess at $10 \mu \mathrm{m}(\mathrm{C} 1653=$ BM Gem and C4595 = V1468 Aql),

- 2 carbon cepheids (C3533 = V553 Cen of CWB-type and period 2.0605 days, and C1622 = RU Cam of CWA-type with period variable from 22.05 to 22.24 days), and the oxygen-rich cepheid RT TrA for comparison (CWB-type with period 1.9461 days),

- C3066 = HD100764, the HC1-star with strong infrared excess, shown without correction (brighter value) and with correction (fainter value),

- AC Her, a carbon-rich variable of RV Tau-type.
The mean values quoted in Table 3 for the fourteen photometric groups of the carbon-rich atmospheres, are also shown as (heavy) starred symbols with dispersion bars. Spanning the 5800-2000 K range, from left to right, they are: HC0 (2 values: see caption), $\mathrm{HC} 1, \mathrm{HC} 2, \mathrm{HC} 3$ and $\mathrm{HC} 4\left(\left\langle M_{\mathrm{bol}}\right\rangle \simeq-3\right.$ at about the same $\left.\log T_{\text {eff }} \simeq 3.6\right), \mathrm{HC} 5, \mathrm{CV} 1, \mathrm{CV} 2, \mathrm{CV} 3, \mathrm{SCV}$ $\left(\left\langle M_{\text {bol }}\right\rangle \simeq-5.8\right)$ and CV4 (at about the same $\left.\log T_{\text {eff }} \simeq 3.445\right)$, CV5, CV6 and CV7. Oxygen-rich and BaII stars are considered separately.

\subsection{Theoretical evolutionary tracks and mass range}

Detailed accounts of the evolution of red giants can be found in reviews like Iben \& Renzini (1983) and more recently Busso et al. (1999). Sketches of evolutionary tracks in the two cases described hereafter, may be found in the latter reference (p. 243). For low- and intermediate-mass stars, the termination of evolution in the red giant portion of the HR diagram, is the asymptotic giant branch (AGB) prior to white dwarf and/or 
planetary nebulae stages. After ascending the red giant branch (RGB), the stars with initial mass $M_{\mathrm{i}} \geq M_{\mathrm{HeF}}$ start the $3 \alpha$ reaction in their central regions $\left({ }^{4} \mathrm{He}\right.$ combustion) and a more or less pronounced loop is executed leftward in the HR diagram, depending on chemical composition and mass: the lower the metallicity, the larger the loop. After core helium exhaustion, the early-AGB (E-AGB) phase is reached, and a second dredgeup experienced. According to models, there is not enough carbon dragged up throughout the envelope, up to the atmosphere, to turn the star into a carbon one. Then higher on the AGB (TP-AGB), the star presents a double shell source surrounding a carbon-oxygen core. It undergoes thermal pulses (TPs; temporal $\mathrm{He}$ shell ignition), experiencing the third dredge-up (TDU). After a more or less large number of TPs, it becomes a carbon star, provided $M_{i} \lesssim 4 M_{\odot}$ since, at larger masses, hot bottom burning (HBB) prevents $\mathrm{C} / \mathrm{O} \geq 1$ to be reached (e.g. Marigo et al. 1999; their Figs. 6 and 7 p. 134). For $M_{\mathrm{i}} \gtrsim 4 M_{\odot}$, $\mathrm{C} / \mathrm{O} \geq 1$ could however be reached in a small luminosity range. Substantial mass loss may reduce the envelope mass and stop HBB while thermal pulses are still occurring (Frost et al. 1998).

For $M_{\mathrm{i}} \leq M_{\mathrm{HeF}}$, eccentric ignition of helium occurs in a degenerate core leading to a core flash which removes degeneracy, followed by a sudden luminosity decrease. According to current models, no dredge-up is found during those flashes. Such a mixing event could explain low-luminosity carbon stars if it actually occurred. After the flash, central helium burning operates in the core, and after exhaustion, the E-AGB and TPAGB phases are subsequently experienced. The "helium flash mass" is about $M_{\mathrm{HeF}} \simeq 2.1-2.2 M_{\odot}$ in models without convective overshoot, depending on the chemical composition.

The detailed evolution is quite complicated as can be seen from models evolved "all-the-way" from main sequence to AGB (see e.g. Sackmann et al. 1993 for a $1 M_{\odot}$ model). Those models give nearly consistent results up to the E-AGB phase. On the contrary, they diverge in the TP-AGB phase, depending on parameters and codes used. A critical topic is that of the minimum luminosity at which a $\mathrm{C} / \mathrm{O}$ value larger than unity is reached. Varying initial mass, metallicity, dredge-up efficiency, mixing length, eventually adding convective overshoot..., and using different codes with various time and space resolutions, the issue appears as seriously confused (see Marigo et al. 1999). The lowest transition obtained or carbon star formation line (CSFL), corresponds to $M_{\text {bol }} \simeq-3.5$ but -4.0 at least is required in most models. More or less strong and episodic mass loss intervenes during those advanced stages as evidenced by the observations of circumstellar shells around many of those stars (Wallerstein \& Knapp 1998; Schöier \& Olofsson 2001). This phenomenon strongly influences stellar evolution and dissipating the stellar envelope may halt the ascent of the AGB (e.g. Sackmann et al. 1993). Detailed calculations of stellar evolution have been published which assume a fixed rate of mass loss (Steffen et al. 1998).

An alternative to fully-numerical models evolved all the way from the main sequence, are semi-analytical models which use relations calibrated from the former, like the core massluminosity relation and core mass-interpulse period relation. We have adopted the results of Marigo et al. (1996) coupled with the full calculations of Bressan et al. $(Z=0.02,1993)$ and those of Fagotto et al. $(Z=0.008,1994 \mathrm{c})$. The former results are shown in Fig. 8, as light continuous curves for initial masses $0.8,1,2$ and $4 M_{\odot}$, while the latter (heavy dashed curves) are displayed for 1 and $1.9 M_{\odot}$. Those tracks illustrate the shift due to metallicity differences. They cannot describe the full complexity of those evolution phases, but are merely guidelines we adopt here. Taking into account the large dispersions of the observational results $( \pm 0.9 \mathrm{mag}$ on average $)$, they delineate fairly well the loci of the carbon and BaII giants (we estimated intrinsic dispersion on mean values for photometric groups to be about $0.75 \mathrm{mag}$ ). We conclude that the $0.8 M_{\odot} \leq M_{\mathrm{i}} \leq 4.0 M_{\odot}$ range, with probably a large spread in chemical composition, reproduces fairly well the observations. A comparable result $\left(1.0 M_{\odot} \leq M_{\mathrm{i}} \leq 4.0 M_{\odot}\right)$ was already reached by Alksnis et al. (1998), making use of about forty observed HIPPARCOS parallaxes. Their sample is however limited in number and accuracy $\left(0.16 \leq \sigma_{\varpi_{0}} / \varpi_{0} \leq 0.98\right)$, and mean values would then be biased. This kind of range in initial masses is also what is excepted putting together the various subsamples in the pre-HIPPARCOS studies, as obtained from different techniques and data bases (Claussen et al. 1987; Thronson et al. 1987; Zuckerman \& Dyck 1989; Barnbaum et al. 1991). The agreement is also good with studies on individual sources like IRC +10216 . Isotopic ratios observed in the circumstellar envelope point to a low stellar mass, excluding a $5 M_{\odot}$-model, making use of theoretical nucleosynthesis (Kahane et al. 2000).

Returning to Fig. 8, we observe that the RCB variables strongly depart from the above loci and a $\left(3.0 M_{\odot} \leq M_{\mathrm{i}} \leq 6.0 M_{\odot}\right)$ range for $Z=0.02 \mathrm{might}$ be considered, or alternatively less massive models with lower metallicities and thus larger leftward loops. The chemical peculiarities of at least some RCB variables and cooler non-variable hydrogen-deficient analogues, i.e. carbon stars named $\mathrm{HdC}$, are best reproduced by "born-again" low-mass giants (Schönberner 1979; Herwig et al. 1999 and references therein). Born-again stars experience a last thermal pulse while approaching the white dwarf zone in the HR diagram, and thus return into the red-giant region, with a tiny envelope.

\subsection{The Ball giants}

The BaII giants populate the whole domain $1.1 \geq M_{\mathrm{bol}} \geq-2.9$ for $3.6 \leq \log \left(T_{\text {eff }}\right) \leq 3.72$, but the largest concentration is found between 1.2 and -0.6 , centered at about $M_{\mathrm{bol}} \simeq 0.3$. With $B C_{V} \simeq-0.3$ to -0.8 , depending on the effective temperature, it corresponds to $0.6 \leq M_{V} \leq 1.1$. Those values point to the luminosity class IIIb as revised by Keenan \& Barnbaum (1999), henceforth centered on the clump, an obvious concentration in the HR diagrams of some old open clusters. The attributing to classes III (or IIIab) and then to class IIIa is somewhat more difficult. A second concentration close to $M_{\mathrm{bol}} \simeq-2.5$, i.e. $M_{\mathrm{V}} \simeq-2.0$, is corresponding to class II (bright giants).

It can be seen from Fig. 8, that the BaII giants of class III are located between the tracks for stars of initial masses in the range 1 to $4-5 M_{\odot}$, in the RGB region and/or leftward loops of central He-burning. The brighter BaII giants of class II seem to 
be in the E-AGB phase. For $Z=0.02$, many BaII stars could have initial masses in the $1.5-2.5 M_{\odot}$ range. From radial velocity variations, it has been shown that those stars are members of binary stars, whose former primary then on the AGB, is now a white dwarf (McClure et al. 1980; Jorissen \& Boffin 1992). Heavy elements enrichment ( $\mathrm{Sr}, \mathrm{Ba})$ is thus the consequence of mass transfer in the binary system. This is also the case of "extrinsic" S stars (Van Eck et al. 1998) and possibly of the CH stars (McClure \& Woodsworth 1990).

\subsection{The HC-giants}

Considering the evolutionary tracks of Fig. 8, it appears that the hot carbon stars (HC-groups, mostly R stars) are located in the RGB and/or He burning loops, and also the E-AGB, like the BaII stars. The former are however brighter by about 1 mag than the latter, on average. The cool carbon variables (CVgroups, mostly $\mathrm{N}$ variables: Sect. 7.5 ) seem essentially located in the TP-AGB region. The case of the HC5-group, located at the junction $\left(M_{\mathrm{bol}} \simeq-3.4\right)$ is not clear as already noticed on the grounds of space distributions and velocities (Paper II). The latter studies have shown that most HC-stars are members of the thick disk population, while the CV-stars are old (thin) disk members. Amongst the former stars, are found the $\mathrm{CH}$ stars which are members of the spheroid. Despite the aspect of Fig. 8, most of the HC-giants are not the progenitors of the $\mathrm{CV}$-ones. The age of the thick disk certainly lies between 9 and 12 Gyr, with 11 Gyr usually adopted. From calculations of Fagotto et al. (1994a, 1994b) the corresponding initial masses range from 0.88 to $0.98 M_{\odot}$ for $Z=0.0004$ to $Z=0.008$.

The low luminosity of HC-stars $\left(\left\langle M_{\mathrm{bol}} \gtrsim-3.4\right\rangle\right)$ makes their carbon enrichment not explained by TDU on TPAGB. It occurs, at least in models, at higher luminosities. Alternative possibilities will be discussed in Sect. 6 of Paper IV.

\subsection{The CV-giants}

\subsubsection{The TDU on TP-AGB}

We consider now the luminous CV-stars. They are essentially $N$ variables and late $\mathrm{R}$ stars which can be explained by the third dredge-up (TDU). This phenomenon does intervene when the convective envelope is able to penetrate the region located between the two shells (He-burning inside, H-burning outside, alternatively ignited) where lies the stellar material which just experienced He-burning during the flash (Iben \& Renzini 1983). Busso et al. (1999) summarize recent versions of the scenario, with a better knowledge of the fate of ${ }^{13} \mathrm{C}$-pockets formed by diffusive and semiconvective mixing at dredge-up. These pockets are the site where the s-elements observed in many CV-stars are produced. A fraction $\Delta M_{\text {dredge }}$ of the intershell material is convected to the surface, which is polluted in $\mathrm{He}, \mathrm{C}$ and $\mathrm{O}$, and traces of heavy elements from the s-process (Lattanzio \& Boothroyd 1997; Busso et al. 2001). Through subsequent mass loss, low- and intermediate-mass stars contribute to the chemical enrichment of the interstellar medium (e.g. Marigo 2001).
However, most numerical models evolved all the way from the main sequence up to the TP-AGB, either neither reach $\mathrm{C} / \mathrm{O} \geq 1$ or become carbon stars at very high luminosities corresponding to $M_{\text {bol }} \simeq-5$ to -6.5 (see Marigo et al. 1999 , their Table 1 p. 125 , for a summary). This was the reason for developing semi-analytical models whose parameters are adjusted to fit the observations (Marigo et al. 1996; Marigo et al. 1999), like

- the minimum core mass $M_{\mathrm{c}}^{\mathrm{min}}$ allowing TDU appearance,

- the TDU efficiency $\lambda=\Delta M_{\text {dredge }} / \Delta M_{\mathrm{c}}$, where $\Delta M_{\mathrm{c}}$ is the downward convective penetration,

- the chemical composition of the intershell convective zone.

They include analytical laws deduced from numerical models evolved all the way from the main sequence, and initial conditions taken at the onset of the first pulse from those models. The TP-AGB portions of the tracks of Fig. 8 were obtained from this method (Marigo et al. 1996). Actually, the used $M_{\mathrm{c}}-L$ relation is only representative of the bright terminal portion of the inter-pulse phase. It explains the steady high luminosity of those tracks, as opposed to the sudden drops of complete numerical models such as those of Sackmann et al. (1993).

The concept of $M_{\mathrm{c}}^{\mathrm{min}}$, a function of mass and metallicity (say $Z$ ), is abandoned in the improved version of Marigo et al. (1999). They claim they satisfactorily reproduce the LFs of LMC $(Z=0.008)$ and SMC $(Z=0.004)$. Their minimum luminosity for carbon star production is at about $M_{\text {bol }} \simeq-4$ in both cases (their Figs. 6 and 7 p. 134). However, due to two quiescence phases intervened in star formation history of the LMC clusters, one could not establish the lower and upper limit to carbon star masses in the LMC (Marigo et al. 1996).

\subsubsection{The inferred mass range}

Taking into account the errors on parallaxes, the locus of CVstars in Fig. 8 is nearly bounded downward by the $M=$ $0.8-1 M_{\odot}$ tracks at $Z=0.02$, which is not surprising. This is an old (thin) disk population and the evolution time needed by such low mass stars to reach the red giant region, is at least $\tau \simeq 10.3 \times 10^{9}$ years (Bressan et al. 1993). This is about the age of the Galactic old disk $\left(10-11 \times 10^{9}\right.$ years $)$. The tracks and evolution time are however dependent on the chemical composition $(X, Y, Z)$. For $Z=0.008$, the $M=1 M_{\odot}$ track is shifted toward higher luminosities and temperatures. The evolution time shortens to $\tau \simeq 8.5 \times 10^{9}$ years, and to $\tau \simeq 7.1 \times 10^{9}$ years for $Z=0.004$. There is probably a large scatter around a mean age-metallicity relationship, due to local inhomogeneities. We conclude that the distribution of Fig. 8 corresponds to an inferior limit for initial masses of $0.85-1.1 M_{\odot}$ with various metallicities and an evolution time to the red giant region of 8-11 Gyr, depending on $Z$-values.

The upper edge of the locus of CV-stars in Fig. 8 is approximately the $M=4 M_{\odot}$ track at $Z=0.02$. It corresponds to a lower limit of $1.5 \times 10^{8}$ years for the evolution time of those objects. This upper limit in initial masses decreases if lower metallicities are assumed. Apart from RCB variables and HdC stars classified $\mathrm{HC} 0$ or $\mathrm{HC} 1$ with $T_{\text {eff }} \simeq 4500-7000 \mathrm{~K}$ 
and $-4 \leq M_{\text {bol }} \leq-2$, a few stars are located on the left of this limit, namely

\section{- SC stars classified SCV and oxygen-rich red giants, \\ - some very cool and luminous carbon stars classified CV6 or CV7.}

Those stars may correspond to $M=4-6 M_{\odot}$ evolutionary tracks $(Z=0.02)$ not shown in Fig. 8. Recently, very bright carbon stars $\left(M_{\text {bol }} \simeq-6.8\right)$ surrounded by thick circumstellar shells were discovered in the LMC (van Loon et al. 1998, 1999). They correspond to our brightest Galactic CV7-stars (up to $M_{\text {bol }} \simeq-7$ ), but unfortunately, very few parallaxes are available for them. They probably represent a minority amongst CVstars. According to Figs. 6 and 7 of Marigo et al. (1999), they may correspond to "obscured C-stars" with $M_{\mathrm{i}} \geq 4-4.5 M_{\odot}$, which skip from oxygen-rich to carbon-rich objects, and then backwards.

The six oxygen-rich giants (neither RCB variables nor BaII stars) and four SC stars (classified SCV) in our sample fall either above the $M=4 M_{\odot}$ track or slightly below it, but within \pm 0.9 mag. It is confirmed that the upper part of the HR diagram is populated with oxygen-rich giants or supergiants, and OH-IR LPVs. This is also the region of intrinsic S stars (see Van Eck et al. 1998), which are Tc-enriched TP-AGB stars (see Sect. 7.5.3 for Tc-rich carbon stars). The admitted explanation is hot bottom burning (HBB) in stars with $M_{\mathrm{i}} \geq$ 4-5 $M_{\odot}$ : the convective envelope spreads in depth until high temperature regions are reached where hydrogen combustion via the CNO cycle is going on (e.g. Marigo et al. 1998). Then ${ }^{12} \mathrm{C}$ precedently synthesized by the $3 \alpha$ reaction is burnt into ${ }^{14} \mathrm{~N}$. The main consequences are a decrease of the $\mathrm{C} / \mathrm{O}$ ratio which can become again less than unity, and a lowering of the ${ }^{12} \mathrm{C} /{ }^{13} \mathrm{C}$-ratio. Making use of the $M_{\mathrm{c}}-L$ relationship of Wagenhuber \& Groenewegen (1998), the calculations have been refined (Marigo 1998; Figs. 6 and 7 of Marigo et al. 1999 may also be used). Strong mass loss might promptly reduce the envelope mass in such a way that HBB could stop while TDU is still going on. Frost et al. (1998) thus obtained models of carbon stars with optically thick wind at the tip of TP-AGB, and luminosities comparable to those of the obscured LMC C-stars of van Loon et al. (1998, 1999), and of our brightest CV7-stars.

We note that the carbon stars with strong silicate-type excesses in the infrared (BM Gem and V1468 Aql) fall exactly in the middle of the loci at HC5-CV1 $\left(T_{\text {eff }} \simeq 3500-3300 \mathrm{~K}\right)$, far below the oxygen-rich limit roughly identified with the $M=4 M_{\odot}$ evolutionary track.

\subsubsection{Tc and ${ }^{12} \mathrm{C} /{ }^{13} \mathrm{C}$-ratio}

A relation was established between the $\mathrm{CV}$-classification, and thus effective temperature, and the $\mathrm{C} / \mathrm{O}$ ratio (Paper I; Sect. 17 and Fig. 16): mean ratios and dispersions increase along the CV2-CV6 sequence, i.e. for decreasing effective temperatures and increasing luminosities. Then we checked for possible correlation of our data with the ${ }^{12} \mathrm{C} /{ }^{13} \mathrm{C}$-ratio: the carbon stars with low ratios prove to be rather uniformly distributed in the carbon star locus of Fig. 8 in both the HC- and CV-portions.
The so-called J-stars ( ${ }^{13} \mathrm{C}$-rich) show no preference concerning effective temperatures and luminosities. The ${ }^{13} \mathrm{C}$ producing neutrons must be created inside He-rich, partly He-burning regions (He shell), not in the envelope. Thus the J-stars cannot be explained by an extra-mixing, i.e. cool bottom processing (CBP) on the AGB (Nollett et al. 2001, 2002) because this does not account for other abundances, and would invariably lead to s-element rich stars, while the J-stars are not s-process rich. The ${ }^{13} \mathrm{C}$ observed in J-stars must be produced by some extra-mixing at relatively low temperatures, in phases that never experienced conditions suitable for producing neutrons.

We finally show in Fig. 9, a new version of the same HR diagram, but restricted to the carbon giants. The practically vertical separation line between the loci of HC- and CV-stars, is located at $\log T_{\text {eff }} \simeq 3.52$ i.e. $T_{\text {eff }} \simeq 3300 \mathrm{~K}$. It is very close to the theoretical curve produced by Scalo (1976) for the onset of helium shell flashes for a Galactic disk composition. Following Westerlund et al. (1995; Fig. 4), this is also the carbon star formation line (CSFL) for the CV-stars which are old (thin) disk stars on TP-AGB. Taking into account the errors on parallaxes and the mean values of Table 3 , we set the lower limit

$M_{\mathrm{bol}} \simeq-3.6 \pm 0.4$

for the majority of the CV-stars which is in satisfactory agreement with $M_{\text {bol }} \leq-4.0$ of Marigo et al. (1999). A few CV-giants (including Miras), appear as underluminous $\left(\left\langle M_{\text {bol }}\right\rangle \simeq-3.3\right.$ to -3.6$)$ as always noted by Bergeat et al. (1998) in a period-luminosity diagram (their Sample 3; similar objects are observed in the LMC). Pulsation masses for those underluminous CV-stars, are systematically found in the 0.5-0.6 $M_{\odot}$ range (Sect. 3.2 of paper IV). The bright CV-stars are expected to be enriched in heavy elements as predicted from TDU. A probing case is that of tecnetium $\left({ }^{99} \mathrm{Tc}\right.$ an unstable isotope with half life $\tau \simeq 2.14 \times 10^{5}$ years). A Tc-rich atmosphere has thus necessarily experienced a recent dredge-up (a few $10^{5}$ years). The Tc-stars among carbon giants are shown as (heavy) starred symbols in Fig. 9. We note that they all are CV-stars. No one is observed in the HC-region of the diagram, leftward of the separation boundary or CSFL for CV-stars. The statement was however made of Tc-lines absent from the spectra of several CV-stars like UU Aur, X Cnc, Y CVn and probably SS Vir, the former two being enriched in s-process elements (Little et al. 1987). On the contrary, Tc seems present in every bright (intrinsic) S star (Van Eck et al. 1998, their Fig. 2 p. 977 to be compared to Fig. 9). However, technetium is best seen from its blue resonance line at $\lambda \simeq 426.0 \mathrm{~nm}$, in a region of the spectrum generally unreachable in very red C-stars, due to a strong depression at $440 \mathrm{~nm}$. Other features like the inter-combination Tc I line at $\lambda \simeq 592.447 \mathrm{~nm}$, are extremely blended and much weaker. It often cannot be seen, which cannot be directly interpreted as inferring that it is not here (Abia et al. 2001). Further investigations are clearly needed before this matter can be settled once and for all. We have determined for Tc-rich carbon giants (Abia et al. 2001, and references therein), a kind of a barycenter ( $n=22$ stars $)$ at $\left\langle C_{\mathrm{L}}\right\rangle \simeq 1.30 \pm 0.45$ i.e. $\left\langle L / L_{\odot}\right\rangle \simeq 5900$, or alternatively

$\left\langle M_{\mathrm{bol}}\right\rangle \simeq-4.7(-4.05 ;-5.6)$ 


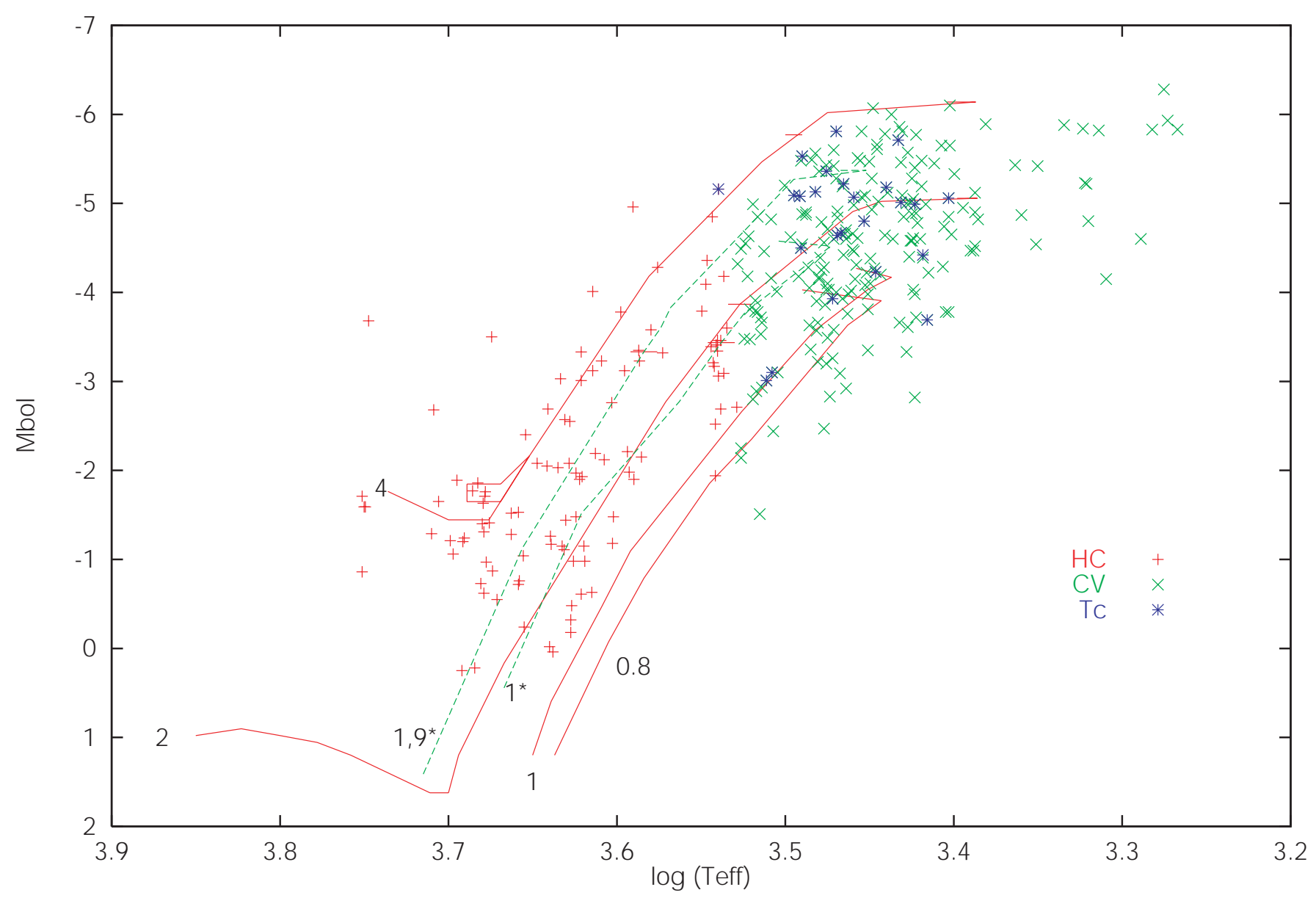

Fig. 9. The HR diagram of about 300 carbon giants. The nearly vertical limit between HC-stars on the left, and CV-stars on the right, is clearly seen. The Tc-rich stars are located in the bright locus of CV-stars which are TP-AGB objects.

which is 1 mag above the lower limit, and

$\left\langle T_{\text {eff }}\right\rangle \simeq(2935 \pm 200) \mathrm{K}$

which correspond to the groups CV3-CV4 in Table 3 . The absence of Tc detection in cooler stars (CV7- and most CV6giants) may be due to insufficient coverage and/or technical difficulties while investigating those overcrowded spectra with many blends. The identification of the cool carbon variables of the CV-groups with TP-AGB objects having recently experienced dredge-up of nuclearly-processed material, makes no doubt on the grounds of the above arguments, even if the interpretation of detailed chemical peculiarities remains difficult.

\subsection{The RCB variables, $\mathrm{HdC}$ giants and carbon-rich Cepheids}

About fifteen RCB variables and hydrogen deficient carbon (HdC) stars are shown in Fig. 8 where they form a leftward protuberance. The plot in Fig. 9 is restricted to stars classified in carbon-rich (HC) groups. Many of those stars are close to the $M=4 M_{\odot}$ track or above it. It is however surprising to find faint RCB variables of $M_{\text {bol }} \geq-2$, since they were believed to be bright giants or even supergiants on the grounds of their spectra. The latter are probably more typical of atmospheric extension and/or mass loss, than of truly large luminosities. We shall see in Sect. 3 of Paper IV that there is no correlation between surface gravity and luminosity in the case of the CV-giants.

The results for a few representative stars as collected from Table 2 available at CDS, are given in Table 5. A correlation is obvious from Fig. 8 and Table 5: the RCB variables classified into oxygen-type groups, with relatively high effective temperatures (about 6000-7000 K and spectral type F), reach $M_{\mathrm{bol}} \simeq-3.5$ to -4.8 , i.e. luminosities similar to those of the brightest $\mathrm{HdC}$ stars like $\mathrm{C} 4247\left(M_{\mathrm{bol}} \simeq-3.7\right)$ and C3606 $\left(M_{\text {bol }} \simeq-3.5\right)$ which are labelled in Fig. 8 . It is the case of $\mathrm{R} \mathrm{CrB}$ and RY Sgr. This is also close to $M_{\text {bol }} \simeq-5.0$ found for the early RCB-variables in the LMC (W Men and HV 5637; a distance modulus of 18.5 was assumed). Then, from $M_{\text {bol }} \simeq-1.5$ to -3.0 , we found some RCB variables which have been included by Stephenson in his catalogue (1989), e.g. $\mathrm{V}$ CrA $=\mathrm{C} 4098$ and $\mathrm{RS}$ Tel $=\mathrm{C} 3982$, as carbon stars, but to whom we attributed oxygen-rich groups in our analyses. At nearly the same luminosities, but with slightly lower effective temperatures, we find a few RCB variables classified as carbon stars by Stephenson, and HC0 to HC3 by us. It is the case of RT Nor $=$ C3687, S Aps $=$ C3562 and SV Sge $=$ C4181.

The transition between the oxygen-type groups and the HCones is thus confirmed at $T_{\text {eff }} \simeq(5600 \pm 200) \mathrm{K}$, as is the case 
Table 5. The results obtained for 11 peculiar stars: 7 RCB variables, 3 cepheids and AC Her a RV Tau-type variable. They are selected from Table 2 available at CDS. The photometric group and corresponding effective temperature (Cols. 3 and 5), the extinction $A_{J} \simeq 0.87 E(B-V)$ (Col. 4), were derived from our SED analyses (the star-symbol denotes a circumstellar contribution to the extinction). The obtained absolute bolometric magnitudes are quoted in Col. 6 and comments in Col. 7 refer to periods in days, phases and eventual label $\mathrm{Hd}$ or $\mathrm{HdC}$ for the hydrogen-deficient stars.

\begin{tabular}{ccccccc}
\hline \hline Name & Type & PhG & $A_{J}$ & $T_{\text {eff }}$ & $M_{\text {bol }}$ & Comments \\
\hline R CrB & RCB & F8sg & 0.0 & 6100 & -3.7 & Hd; max \\
RY Sgr & RCB & F5sg & 0.07 & 6900 & -4.4 & Hd; max \\
V CrA & RCB & F9g & 0.11 & 5800 & -2.3 & max \\
V CrA & RCB & F9g & $0.20^{*}$ & 5800 & -1.7 & int \\
RS Tel & RCB & G0sg & 0.07 & 5800 & -1.4 & $\max$ \\
RS Tel & RCB & G0sg & $0.62 *$ & 5800 & +0.5 & $\min$ \\
RT Nor & RCB & HC0 & 0.28 & 5615 & -1.8 & HdC \\
S Aps & RCB & HC1 & 0.20 & 5115 & -2.5 & HdC \\
SV Sge & RCB & HC3 & 0.40 & 4010 & -2.8 & HdC \\
RT TrA & CWB & F6.5sg & 0.075 & 6500 & -1.0 & $1.9461 \mathrm{~d} ; 0.0$ \\
RT TrA & CWB & G0sg & 0.070 & 5850 & -0.8 & 0.2 \\
RT TrA & CWB & G2sg & 0.090 & 5200 & -0.6 & $0.6(\min )$ \\
RT TrA & CWB & F8sg & 0.097 & 6100 & -0.75 & 0.8 \\
V553 Cen & CWB & F8g & 0.07 & 6150 & -1.3 & $2.0605 \mathrm{~d} ; \max$ \\
V553 Cen & CWB & G1g & 0.07 & 5650 & -1.0 & $\min$ \\
RU Cam & CWA & HC1 & 0.03 & 5215 & -1.8 & $22.05 \mathrm{~d} ;$ HdC \\
AC Her & RVa & G0g & 0.17 & 5850 & -2.3 & $75.01 \mathrm{~d} ; 0.66$ \\
\hline
\end{tabular}

for non-variable stars. The variations of the RCB stars seem to be not intrinsically photospheric in origin, but rather atmospheric and/or circumstellar, as shown by the increase of extinction at phases outside maxima (see Bergeat et al. 1999 and references therein). The existence of two classes of RCB variables with respectively $\left\langle M_{\text {bol }}\right\rangle \simeq-3.8$ and $\left\langle M_{\text {bol }}\right\rangle \simeq-2.0$, or alternatively of a continuous distribution from -4 to -1 , can be invoked. Those results need to be considered with caution since they are based on very few stars, with data of limited accuracy.

Three cepheids are included in our sample, two of which $($ RU Cam $=$ C1622 and V553 Cen = C3533) were catalogued by Stephenson (1989), and not the third, namely RT TrA. They all are classified CW, i.e. they are members of a Population II group including the pulsating cepheid-like variables of the W Vir and BL Her types. The mean absolute bolometric magnitudes of the CW-stars spread from -0.15 to -2.45 with effective temperatures ranging from 6600 down to 5250 $\mathrm{K}$ (Hall 2000). The results of Table 5 are in good agreement with those values. Making use of the pulsation constant $Q$ as quoted by Hall (2000), a mean pulsation mass of $M_{\mathrm{p}} \simeq 0.57 M_{\odot}$ is found for those three stars, in good agreement with the $0.6 M_{\odot}$ quoted in Hall's table. We find here the same sequence for decreasing effective temperatures, as that found for RCB variables: early oxygen-types not in Stephenson's catalogue, oxygen-types in the catalogue, and carbon HC-types. The data is compatible with a second transition still located at about $5600 \mathrm{~K}$. As is well-known, the variations of those cepheids are clearly photospheric in origin, with pulsations character- ized by large changes in effective temperature and photometric group. The associated change in luminosity amounts typically to $\Delta M_{\text {bol }} \simeq 0.3$ to 0.4 .

The carbon-rich star AC Her, classified as a variable of the RV Tauri type, was studied in some detail by Bergeat et al. (1999 and references therein). The luminosity found here $\left(M_{\mathrm{bol}} \simeq-2.3\right)$ places it in the RCB-range, but it is brighter than the above $\mathrm{CW}$-variables.

\section{Summary of the results}

Four bolometric corrections were calibrated against three color indices for 375 carbon and $\mathrm{Ba}$ II giants and related objects. The mean relations are tabulated in the Appendix. They were selected by trial and error as the more efficient ones, and used to derive the bolometric magnitudes of 81 HIPPARCOS carbon stars and related objects. The coefficients $C_{\mathrm{R}}$ and $C_{\mathrm{L}}$ were defined, which are proportional to the true parallaxes, and are intended for statistical purposes. They allow to estimate unbiased mean photospheric radii and mean luminosities respectively. The relations between the photometric mean coefficient $\langle k\rangle^{1 / 2}$ and the true parallax evaluated from HIPPARCOS astrometry (Knapik et al. 1998; Paper II) were studied and shown to be consistent with the existence of a range of photospheric radii for every photometric group. The data in Table 1 illustrates the increase of the photospheric radius along the sequence of photometric groups from $\mathrm{HC} 1$ to $\mathrm{HC} 5$, and then from $\mathrm{CV} 1$ to $\mathrm{CV} 6$ (the extreme groups $\mathrm{HC} 0$ and $\mathrm{CV} 7$ are poorly-documented).

The above-described work completes the study of Paper I, and the resulting data can be found in Table 2 (only available at CDS), with 507 entries corresponding to the analyzed SEDs. This is a corrected, augmented and updated version of Table 10 of Paper I. Effective temperatures, interstellar (and eventually circumstellar) extinctions, classification in our photometric groups, bolometric magnitudes and comments are quoted there. For about 370 objects, absolute bolometric magnitudes estimated from true parallaxes are also given. This data base was essentially intended for statistical use. Mean data was provided for the various photometric groups (Table 3), and then calculated for five variability classes and quoted in Table 4.

The luminosity function (LF) of our sample of carbon giants (MW), was constructed and compared to the LFs of carbon stars in the Milky Way Bulge (MWB), LMC and SMC (Figs. 5, 6 and 7). It displays two maxima which correspond to components of two distinct populations identified in Paper II. The HR diagram of nearly 370 Galactic carbon and BaII giants, and related objects was constructed (Fig. 8), and explored. The localization of ${ }^{99} \mathrm{Tc}$ stars within the (bright) CV-stars was then confirmed and discussed (Fig. 9). On the contrary, ${ }^{13} \mathrm{C}$-rich (J) stars were found nearly uniformly distributed.

A full discussion and conclusions will be given at the end of the companion article (Paper IV), attempting a synthesis of the results from Papers I, II, III and IV.

Acknowledgements. Valuable suggestions from the referee Dr. Maurizio Busso are gratefully acknowledged. We are especially indebted to him for having pointed out some weaknesses and misunderstandings withdrawn from the present account of predictions from theoretical models. 


\section{Appendix}

Table A.1. The adopted mean relations of bolometric corrections vs. intrinsic color indices from the data of 375 carbon stars and related objects.

\begin{tabular}{|c|c|c|c|c|c|c|c|c|c|c|}
\hline$[\mathrm{V}-[1,08]]_{0}$ & $\mathrm{CB}_{[0,78]}$ & $\mathrm{CB}_{[1,08]}$ & $\mathrm{CB}_{\mathrm{H}}$ & $\mathrm{CB}_{\mathrm{K}}$ & {$[[1,08]-\mathrm{K}]_{0}$} & $\mathrm{CB}_{\mathrm{H}}$ & $\mathrm{CB}_{\mathrm{K}}$ & $(\mathrm{J}-\mathrm{K})_{0}$ & $\mathrm{CB}_{\mathrm{H}}$ & $\mathrm{CB}_{\mathrm{K}}$ \\
\hline 0.70 & 0.66 & 0.80 & 1.09 & 1.18 & 0.4 & 1.0 & 1.2 & 0.1 & & 0.94 \\
\hline 0.80 & 0.67 & 0.85 & 1.23 & 1.27 & 0.5 & 1.21 & 1.41 & 0.2 & 0.94 & 1.14 \\
\hline 0.90 & 0.68 & 0.90 & 1.36 & 1.35 & 0.6 & 1.46 & 1.60 & 0.3 & 1.24 & 1.35 \\
\hline 1.00 & 0.69 & 0.94 & 1.46 & 1.43 & 0.7 & 1.64 & 1.78 & 0.4 & 1.48 & 1.55 \\
\hline 1.10 & 0.71 & 0.96 & 1.57 & 1.53 & 0.8 & 1.80 & 1.95 & 0.5 & 1.67 & 1.76 \\
\hline 1.20 & 0.72 & 1.02 & 1.66 & 1.62 & 0.9 & 1.96 & 2.12 & 0.6 & 1.84 & 1.93 \\
\hline 1.30 & 0.74 & 1.05 & 1.75 & 1.70 & 1.0 & 2.11 & 2.26 & 0.7 & 2.00 & 2.12 \\
\hline 1.40 & 0.75 & 1.10 & 1.83 & 1.80 & 1.1 & 2.23 & 2.40 & 0.8 & 2.13 & 2.26 \\
\hline 1.50 & 0.75 & 1.14 & 1.89 & 1.87 & 1.2 & 2.31 & 2.54 & 0.9 & 2.26 & 2.41 \\
\hline 1.60 & 0.76 & 1.19 & 1.94 & 1.94 & 1.3 & 2.39 & 2.66 & 1.0 & 2.38 & 2.57 \\
\hline 1.70 & 0.76 & 1.22 & 1.99 & 2.03 & 1.4 & 2.46 & 2.78 & 1.1 & 2.48 & 2.71 \\
\hline 1.80 & 0.77 & 1.26 & 2.03 & 2.11 & 1.5 & 2.51 & 2.88 & 1.2 & 2.55 & 2.84 \\
\hline 1.90 & 0.76 & 1.29 & 2.08 & 2.20 & 1.6 & 2.55 & 2.96 & 1.3 & 2.58 & 2.93 \\
\hline 2.00 & 0.76 & 1.29 & 2.12 & 2.25 & 1.7 & 2.58 & 3.01 & 1.4 & 2.59 & 3.00 \\
\hline 2.10 & 0.73 & 1.28 & 2.16 & 2.32 & 1.8 & 2.57 & 3.05 & 1.5 & 2.57 & 3.03 \\
\hline 2.20 & 0.72 & 1.26 & 2.20 & 2.37 & 1.9 & 2.57 & 3.10 & 1.6 & 2.55 & 3.06 \\
\hline 2.30 & 0.69 & 1.25 & 2.24 & 2.42 & 2.0 & 2.56 & 3.15 & 1.7 & 2.56 & 3.14 \\
\hline 2.40 & 0.66 & 1.25 & 2.27 & 2.48 & 2.1 & 2.55 & 3.17 & 1.8 & 2.57 & 3.18 \\
\hline 2.50 & 0.63 & 1.25 & 2.31 & 2.53 & 2.2 & 2.53 & 3.21 & 1.9 & 2.57 & 3.21 \\
\hline 2.60 & 0.59 & 1.25 & 2.35 & 2.59 & 2.3 & 2.51 & 3.22 & 2.0 & 2.49 & 3.25 \\
\hline 2.70 & 0.55 & 1.26 & 2.38 & 2.64 & 2.4 & 2.50 & 3.23 & 2.1 & 2.42 & 3.25 \\
\hline 2.80 & 0.51 & 1.27 & 2.41 & 2.69 & 2.5 & 2.46 & 3.23 & 2.2 & 2.36 & 3.25 \\
\hline 2.90 & 0.48 & 1.28 & 2.44 & 2.73 & 2.6 & 2.41 & 3.23 & 2.3 & 2.29 & 3.25 \\
\hline 3.00 & 0.44 & 1.30 & 2.48 & 2.78 & 2.7 & 2.36 & 3.22 & 2.4 & 2.23 & 3.25 \\
\hline 3.10 & 0.41 & 1.31 & 2.51 & 2.84 & 2.8 & 2.28 & 3.20 & 2.5 & 2.15 & 3.24 \\
\hline 3.20 & 0.38 & 1.32 & 2.53 & 2.88 & 2.9 & 2.19 & 3.18 & 2.6 & 2.07 & 3.22 \\
\hline 3.30 & 0.34 & 1.34 & 2.56 & 2.93 & 3.0 & 2.08 & 3.17 & 2.7 & 1.99 & 3.20 \\
\hline 3.40 & 0.30 & 1.35 & 2.57 & 2.98 & 3.1 & 1.95 & 3.15 & 2.8 & 1.92 & 3.13 \\
\hline 3.50 & 0.25 & 1.33 & 2.57 & 3.00 & 3.2 & 1.84 & 3.12 & 2.9 & 1.85 & 3.06 \\
\hline 3.60 & 0.21 & 1.31 & 2.58 & 3.02 & 3.3 & 1.71 & 3.09 & 3.0 & 1.78 & 2.97 \\
\hline 3.70 & 0.18 & 1.28 & 2.58 & 3.03 & 3.4 & 1.57 & 3.07 & 3.1 & 1.7 & 2.9 \\
\hline 3.80 & 0.16 & 1.27 & 2.57 & 3.06 & 3.5 & 1.45 & 3.04 & & & \\
\hline 3.90 & 0.14 & 1.25 & 2.57 & 3.06 & & & & & & \\
\hline 4.00 & 0.11 & 1.23 & 2.56 & 3.11 & & & & & & \\
\hline 4.10 & 0.09 & 1.19 & 2.56 & 3.12 & & & & & & \\
\hline 4.20 & 0.07 & 1.14 & 2.55 & 3.15 & & & & & & \\
\hline 4.30 & 0.00 & 1.07 & 2.54 & 3.16 & & & & & & \\
\hline 4.40 & -0.06 & 0.99 & 2.53 & 3.18 & & & & & & \\
\hline 4.50 & -0.13 & 0.85 & 2.52 & 3.22 & & & & & & \\
\hline 4.60 & -0.27 & 0.71 & 2.50 & 3.23 & & & & & & \\
\hline 4.70 & -0.43 & 0.59 & 2.47 & 3.23 & & & & & & \\
\hline 4.80 & -0.62 & 0.44 & 2.42 & 3.23 & & & & & & \\
\hline 4.90 & -0.91 & 0.27 & 2.34 & 3.23 & & & & & & \\
\hline 5.00 & -1.51 & 0.00 & 2.19 & 3.22 & & & & & & \\
\hline 5.10 & -2.3 & -0.38 & 1.52 & 3.22 & & & & & & \\
\hline 5.20 & & & & 3.21 & & & & & & \\
\hline 5.30 & & & & 3.20 & & & & & & \\
\hline 5.40 & & & & 3.19 & & & & & & \\
\hline 5.50 & & & & 3.18 & & & & & & \\
\hline
\end{tabular}

\section{References}

Abia, C., Busso, M., Gallino, R., et al. 2001, ApJ, 559, 1117

Alves, D. R. 2000, ApJ, 539, 732

Alksne, Z., Alksnis, A., \& Dzervitis, U. 1991, Properties of Galactic Carbon Stars (Orbit Book Company)

Alksnis, A., Balklavs, A., Dzervitis, U., \& Eglitis, I. 1998, A\&A, 338, 209

Azzopardi, M. 1993, in New Aspects of Magellanic Cloud Research, ed. B. Baschek, G. Klare, \& J. Lequeux (Springer-Verlag, Berlin), 86

Azzopardi, M. 1999a, Ap\&SS, 265, 291
Azzopardi, M. 1999b, in New Views of the Magellanic Clouds, ed. Y.H. Chu, N. B. Suntzeff, J. E. Hesser, \& D. A. Bohlender (Astron. Soc. Pac. publish.), IAU Symp., 190, 324

Azzopardi, M. 2000, in The Carbon Star Phenomenon, ed. R. F. Wing (Kluwer Acad. Publish., Dordrecht), IAU Symp., 177, 51

Azzopardi, M., Rebeirot, E., Lequeux, J., \& Westerlund, B. E. 1991, A\&AS, 88, 265

Azzopardi, M., Muratorio, G., Breysacher, J., \& Westerlund, B. E. 1999, in The Stellar Content of Local Group Galaxies, ed. P. Whitelock, \& R. Cannon (Astron. Soc. Pac. publish.), IAU Symp., 192, 144

Barnbaum, C., Kastner, J. H., \& Zuckerman, B. 1991, AJ, 102, 289 
Baumert, J. H. 1972, The Ohio State university, unpublished thesis

Bergeat, J., \& Knapik, A. 1997, A\&A, 321, L9

Bergeat, J., Knapik, A., \& Rutily, B. 1998, A\&A, 332, L53

Bergeat, J., Knapik, A., \& Rutily, B. 1999, A\&A, 342, 773

Bergeat, J., Knapik, A., \& Rutily, B. 2001, A\&A, 369, 178 (Paper I)

Bergeat, J., Knapik, A., \& Rutily, B. 2002a, A\&A, 385, 94 (Paper II)

Bergeat, J., Knapik, A., \& Rutily, B. 2002b, A\&A, 390, 987 (Paper IV)

Bessell, M. S., Wood, P. R., \& Lloyd Evans, T. 1981, Proc. ASA, 2, 291

Bessell, M. S., Wood, P. R., \& Lloyd Evans, T. 1983, MNRAS, 202, 59

Bressan, A., Fagotto, F., Bertelli, G., \& Chiosi, C. 1993, A\&AS, 100, 647

Brewer, J. P., Richer, H. B., \& Crabtree, D. R. 1995, AJ, 109, 2480

Busso, M., Gallino, R., \& Wasserburg, G. J. 1999, ARA\&A, 37, 239

Busso, M., Gallino, R., Lambert, D. L., Travaglio, C., \& Smith, V. V. 2001, ApJ, 557, 802

Claussen, M. J., Kleinmann, S. G., Joyce, R. R., \& Jura, M. 1987, ApJS, 65, 385

Costa, E., \& Frogel, J. A. 1996, AJ, 112, 2607

Dean, C. A. 1976, AJ, 81, 364

ESA 1995, Future Possibilities for Astrometry in Space, a joint RGOESA Workshop at Cambridge, UK, 19-21 June 1995, ESA SP379

ESA 1997, The HIPPARCOS Catalogue, ESA SP-1200 (ESA)

Fagotto, F., Bressan, A., Bertelli, G., \& Chiosi, C. 1994a, A\&AS, 104, 365

Fagotto, F., Bressan, A., Bertelli, G., \& Chiosi, C. 1994b, A\&AS, 105, 29

Fagotto, F., Bressan, A., Bertelli, G., \& Chiosi, C. 1994c, A\&AS, 105, 39

Feast, M. W., \& Whitelock, P. A. 1992, MNRAS, 259, 6

Frost, C. A., Cannon, R. C., Lattanzio, J. C., Wood, P. R., \& Forestini, M. 1998, A\&A, 332, L17

Frogel, J. A., Persson, S. E., \& Cohen, J. G. 1980, ApJ, 239, 495

Groenewegen, M. A. T. 1998, In ISO's View on Stellar Evolution. Conf. Proc., ed. L. B. F. M. Waters, C. Waelkens, K. A. van der Hucht, \& P. A. Zaal (Noordwijkerhout, The Netherlands. Kluwer \& Academic Publ. Dordrecht), 379

Hall, D. A. 2000, In Allen's Astrophysical Quantities, ed. A. N. Cox, fourth edition (AIP Press, Springer), 400

Han, Z., Eggleton, P. P., Podsiadlowski, P., \& Tout, C. A. 1995, MNRAS, 277, 1443

Hartwick, F. D. A., \& Cowley, A. P. 1985, AJ, 90, 2244

Hartwick, F. D. A., \& Cowley, A. P. 1988, ApJ, 334, 135

Herwig, F., Blöcker, T., Langer, N., \& Driebe, T. 1999, A\&A, 349, L5

Iben, I., \& Renzini, A. 1983, ARA\&A, 21, 27

IRAS Point Source Catalogue, version 2, 1988, Joint IRAS Science Working Group, vol. 2 to 6, NASA RP-1190 (Washington D.C.: U.S. Government Printing Office) (IRAS)

Jorissen, A., \& Boffin, H. M. J. 1992, Binaries as tracers of stellar formation (Cambridge University Press)

Kahane, C., Dufour, E., Busso, M., et al. 2000, A\&A, 357, 669

Keenan, P. C., \& Barnbaum, C. 1999, ApJ, 518, 859

Kholopov, P. N., Samus, N. N., Frolov, M. S., et al. 1985, General Catalogue of Variable Stars (Nauka Publishing House, Moscow) (GCVS). New edition as an electronic version at the Sternberg Institute in Moskow (ftp sai.msu.su with anonymous as user and email-address as pw).

Knapik, A. 1999, Ph.D. Thesis, Université Claude Bernard - Lyon 1

Knapik, A., \& Bergeat, J. 1997, A\&A, 321, 236

Knapik, A., Bergeat, J., \& Rutily, B. 1998, A\&A, 334, 545

Knapik, A., Bergeat, J., \& Rutily, B. 1999, A\&A, 344, 263
Knapp, G., Pourbaix, D., \& Jorissen, A. 2001, A\&A, 371, 222

Lattanzio, J. C., \& Boothroyd, A. I. 1997, in AIP Conf. Proc., 402, ed.

T. Bernatowitz, \& E. Zimer (AIP: Woodbury, NY), 85

Little, S. J., Little-Marenin, I. R., \& Bauer, W. H. 1987, AJ, 94, 901

Lutz, T. E., \& Kelker, D. H. 1973, PASP, 85, 573 (LK)

Malmquist, K. G. 1924, Medd. Lund Astron. Obs., Ser. II, No. 32, 64

Malmquist, K. G. 1936, Stockholms Obs. Medd., No. 26

Marigo, P. 1998, A\&A, 340, 463

Marigo, P. 2001, A\&A, 370, 194

Marigo, P., Bressan, A., \& Chiosi, C. 1996, A\&A, 313, 545

Marigo, P., Bressan, A., \& Chiosi, C. 1998, A\&A, 331, 564

Marigo, P., Girardi, L., \& Bressan, A. 1999, A\&A, 344, 123

McClure, R. D. 1984, PASP, 96, 117

McClure, R. D. 1997a, PASP, 109, 256

McClure, R. D., \& Woodsworth, A. W. 1990, ApJ, 352, 709

McClure, R. D., Fletcher, J. M., \& Nemec, J. M. 1980, ApJ, 238, L35

McLeod, N. W. 1947, ApJ, 105, 390

Mennessier, M. O., Luri, X., Figueras, F., et al. 1997, A\&A, 326, 722

Mennessier, M. O., Mowlavi, N., Alvarez, R., \& Luri, X. 2001, A\&A, 374, 968

Neugebauer, G., \& Leighton, R. B. 1969, Two Micron Sky Survey, NASA SP-3047 (TMSS)

Nollett, K. M., Busso, M., \& Wasserburg, G. J. 2001, Eleventh Annual V. M. Goldsmith Conference, May 20-24, 2001, Hot Springs, Virginia, abstract No. 3898

Nollett, K. M., Busso, M., \& Wasserburg, G. J. 2002, ApJ, submitted

Perrin, G., Coudé du Foresto, V., Ridgway, S. T., \& Mariotti, J.-M. 1998, A\&A, 331, 619

Perryman, M. A. C., Lindegren, L., Kovalevsky, J., et al. 1995, A\&A, 304, 69

Pourbaix, D., \& Jorissen, A. 2000, A\&AS, 145, 161

Rebeirot, E., Azzopardi, M., \& Westerlund, B. E. 1993, A\&AS, 97, 603

Rich, R. M. 1989, in The Center of the Galaxy, ed. M. Morris, (Kluwer Academic Publ., Dordrecht), IAU Symp., 136, 63

Richichi, A., Fabbroni, L., Ragland, S., \& Scholz, M. 1999, A\&A, 344, 511

Rossi, S., Beers, T. C., \& Sneden, C. 1999, in The Third Stromlo Symposium: The Galactic Halo, ASP Conf. Ser., 165, 264

Sackmann, I.-J., Boothroyd, A. I., \& Kraemer A. E. 1993, ApJ, 418, 457

Scalo, J. M. 1976, ApJ, 206, 474

Schöier, F. L., \& Olofsson, H. 2001, A\&A, 368, 969

Schönberner, D. 1979, A\&A, 79, 108

Smith, H. Jr., \& Eichhorn, H. 1996, MNRAS, 281, 211

Steffen, M., Szczerba, R., \& Schoenbener, D. 1998, A\&A, 337, 149

Stephenson, C. B. 1989, Pub. of the Warner \& Swasey Obs. 3, No. 2

Straniero, O., Chieffi, A., Limongi, M., et al. 1997, ApJ, 478, 332

Thronson, Jr. H. A., Latter, W. B., Black, J. H., Bally, J., \& Hacking, P. 1987, ApJ, 322, 770

Van Eck, S., Jorissen, A., Udry, S., Mayor, M., \& Pernier, B. 1998, A\&A, 329, 971

van Loon, J. T., Zijlstra, A. A., Whitelock, P. A., et al. 1998, A\&A, 329,169

van Loon, J. T., Groenewegen, M. A. T., De Koter, A., et al. 1999, A\&A, 351, 559

Wagenhuber, J., \& Groenewegen, M. A. T. 1998, A\&A, 340, 183

Wallerstein, G., \& Knapp, G. R. 1998, ARA\&A, 36, 369

Westerlund, B. E., Azzopardi, M., Breysacher, J., \& Rebeirot, E. 1991, A\&A, 244, 367

Westerlund, B. E., Azzopardi, M., Breysacher, J., \& Rebeirot, E. 1992, A\&A, 260, L4

Westerlund, B. E., Azzopardi, M., Breysacher, J., \& Rebeirot, E. 1995, A\&A, 303, 107

Zuckerman, B., Dyck, H. M., \& Claussen, M. J. 1986, ApJ, 304, 401

Zuckerman, B., \& Dyck, H. M. 1989, A\&A, 209, 119 